\title{
Generating functions of bipartite maps on orientable surfaces
}

\author{
Guillaume Chapuy and Wenjie Fang* \\ LIAFA, UMR CNRS 7089, \\ Université Paris-Diderot, \\ Paris Cedex 13, France \\ \{Guillaume.Chapuy, Wenjie.Fang\}@liafa.univ-paris-diderot.fr
}

Submitted: Aug 25, 2015; Accepted: Aug 3, 2016; Published: Aug 19, 2016

Mathematics Subject Classifications: 05A15;05C30

\begin{abstract}
We compute, for each genus $g \geqslant 0$, the generating function $L_{g} \equiv L_{g}\left(t ; p_{1}, p_{2}, \ldots\right)$ of (labelled) bipartite maps on the orientable surface of genus $g$, with control on all face degrees. We exhibit an explicit change of variables such that for each $g$, $L_{g}$ is a rational function in the new variables, computable by an explicit recursion on the genus. The same holds for the generating function $F_{g}$ of rooted bipartite maps. The form of the result is strikingly similar to the Goulden/Jackson/Vakil and Goulden/Guay-Paquet/Novak formulas for the generating functions of classical and monotone Hurwitz numbers respectively, which suggests stronger links between these models. Our result complements recent results of Kazarian and Zograf, who studied the case where the number of faces is bounded, in the equivalent formalism of dessins d'enfants. Our proofs borrow some ideas from Eynard's "topological recursion" that he applied in particular to even-faced maps (unconventionally called "bipartite maps" in his work). However, the present paper requires no previous knowledge of this topic and comes with elementary (complex-analysis-free) proofs written in the perspective of formal power series.
\end{abstract}

\section{Introduction}

A map of genus $g \geqslant 0$ is a graph embedded into the $g$-torus (the sphere with $g$ handles attached), in such a way that the connected components of the complement of the graph

*Both authors acknowledge partial support from Agence Nationale de la Recherche, grant number ANR 12-JS02-001-01 "Cartaplus", and from the City of Paris, grant "Émergences Paris 2013, Combinatoire à Paris". G.C. is currently visiting McGill University, School of Computer Science, Montréal, Canada, and acknowledges partial support from Luc Devroye. W.F. acknowledges hospitality from LaBRI, UMR CNRS 5800, Université Bordeaux-I, France. An extended abstract of this paper was presented at the conference FPSAC'15 in Korea, July 2015. 
are simply connected. See Section 2.1 for complete definitions. The enumeration of maps is a classical topic in combinatorics, motivated both from the beautiful enumerative questions it unveils, and by its many connections with other areas of mathematics, see e.g. [20]. The enumeration of planar maps (when the underlying surface is the sphere) was initiated by Tutte who showed [22] that the generating function $Q_{0}(t)$ of rooted planar maps by the number of edges is an algebraic function given by:

$$
Q_{0}(t)=s(4-s) / 3 \text { where } s=1+3 t s^{2} .
$$

The enumeration of planar maps has since grown into an enormous field of research on its own, out of the scope of this introduction, and we refer to [21] for an introduction and references.

The enumeration of maps on surfaces different from the sphere was pioneered by Walsh and Lehman [23] who considered restricted families of maps such as one-face maps or maps with a few faces. The question of generalising formula (1), that is, to obtain explicit formulas for the generating function of maps on any given surface was solved by Bender and Canfield, who showed in [2] that for each $g \geqslant 1$, the generating function $Q_{g}(t)$ of rooted maps embedded on the $g$-torus (see again Section 2.1 for definitions) is a rational function of the parameter $s$ defined in (1). For example, for the torus, one has $Q_{1}(t)=$ $\frac{1}{3} \frac{s(s-1)^{2}}{(s+2)(s-2)^{2}}$. This deep and important result was the first of a series of rationality results established for generating functions of maps or related combinatorial objects on higher genus surfaces. Gao [15] proved several rationality results for the generating functions of maps with prescribed degrees using a variant of the kernel method (see Remark 3.1 for a comment about this). Later, Goulden, Jackson and Vakil [18] proved a rationality statement for the generating functions of Hurwitz numbers (an algebraic model having many connections with map enumeration) relying on deep algebraic results [12]. More recently, Goulden, Guay-Paquet, and Novak [16] introduced a variant called monotone Hurwitz numbers, for which they proved a rationality statement very similar to the one of [18]. We invite the reader to compare our main result (Theorem 2.1) with [18, Thm. 3.2] and [16, Thm. 1.4] (see also [16, Sec. 1.5]). The analogy between those results is striking and worth further investigation.

In parallel to this story, mathematical physicists have developed considerable tools to attack problems of map enumeration, motivated by their many connections with high energy physics, and notably matrix integrals (see e.g. [20, Chapter 5]). Among them, the topological recursion is a general framework developed by Eynard and his school $[13,14]$ that solves many models related to map enumeration and algebraic geometry in a universal way (see also the historically important paper [1]). In [13, Chap. 3 Sec. 4.5], this technique is applied to the enumeration of maps on surfaces, and leads in particular to a rationality theorem for generating functions of even maps, i.e. maps with faces of even degrees (that are, unconventionally, called "bipartite" maps in those references, although they are not bipartite in the graph-theoretic sense). The proofs in these references use a complex-analytic viewpoint, and are often not easy to read for the pure combinatorialist, especially given the fact that they are published in the mathematical physics literature. 
The main purpose of the present paper is to establish a rationality theorem for bipartite maps, which is a very natural and widely considered model of maps from both the topological and combinatorial viewpoint, see Section 2.1. Our proof recycles two ideas that appeared in the context of the topological recursion, however previous knowledge of the latter is not required, and our proofs rely only on a concrete viewpoint on Tutte equations and on formal power series. We hope to make some key ideas of the methods related to topological recursion more accessible to pure combinatorialists, using a language that enables an easier comparison with the traditional combinatorial approaches. To be precise, the two crucial steps that are directly inspired from the literature on the topological recursion, and that differ from traditional kernel-like methods often used by combinatorialists are Proposition 3.6 and Theorem 3.9. Once these two results are proved (with a formal series viewpoint), an important part of the work deals then with making explicit the auxiliary variables that underlie the rationality statements (the "Greek" variables in Theorem 2.1 below). This is done in Sections 4 and 5. Finally, the proof of the "integration step" needed to prove our statement in the labelled case (Theorem 2.1) from the rooted case (Theorem 2.3) is an ad hoc proof, partly relying on a bijective insight from [9], see Section 6. This approach has the advantage of giving a partial combinatorial interpretation to the absence of logarithm in generating functions of labelled unrooted maps in genus higher than 1 (Theorem 2.1).

Bipartite maps have been considered before in the literature. The first author studied them by bijective methods [9], and obtained rationality statements that are weaker than the ones we obtain with generating functions here. More recently, Kazarian and Zograf [19], using a variant of the topological recursion, proved a polynomiality statement for the generating functions of bipartite maps with finitely many faces (these authors deal with dessins d'enfants rather than bipartite maps, but the two models are equivalent, see [20, Chap. 1]). By contrast, our main result covers the case of arbitrarily many faces, which is more general. Indeed, not only does it prove that each fixed-face generating function is a polynomial in our chosen set of parameters (by a simple derivation), but it also gives very strong information on the mutual dependency of these different generating functions. Note however that [19] keeps track of one more variable (keeping control on the number of vertices of each colour in their expressions). It is probably possible to unify the two results together.

Let us now mention another motivation for the present work. In our way of applying the ideas of the topological recursion, we have tried to remain as close as possible to the "combinatorialist's view" on the Tutte equations and on formal generating functions. We have also tried to formulate the results as explicitly as we could. These choices are not only designed to make the content more accessible to combinatorialists, but we also believe that such an effort is necessary in the hope of, one day, filling the gap between the current understanding of the main approaches to map enumeration. In particular, the algebraic parametrisations (2), (3) that underlie the rationality statements are clearly related to the generating functions of mobiles appearing in the bijective approaches $[7$, 9], and they also appear in the classical approach to planar Tutte-like equations via catalytic variables $[3,6]$, although the links between these approaches remain a mystery. 
Similarly, the "Greek variables" introduced in Theorem 2.1 to parametrise the high-genus generating functions have a clear combinatorial flavor, that we have tried to make as explicit as we could both in the definition we give, and in the way we make them appear in our computations (Section 5). However, we do not know how to relate those variables to the purely combinatorial approaches of [9]. We hope that the explicit results and computations of this paper may serve as a small step towards a future understanding of these connections.

To finish this introduction, and to prevent a misunderstanding, we mention that the generating functions of bipartite maps of all genera can be collected into a grand generating function that is known to be a Tau-function of the KP (and even 2-Toda) hierarchy, see e.g. [17]. This fact does not play any role in the present paper, and we do not know how to use it to study the kind of questions we are interested in here. However, if this was possible, this could lead to recurrence formulas to compute the generating functions that would be more efficient than the ones we obtain here, as was done so far only in the two very special cases of triangulations [17] and bipartite quadrangulations [8].

This paper is organised as follows. In Section 2, we give necessary definitions and notation, and we state the main results (Theorems 2.1 and 2.3). In Section 3 we write the Tutte/loop equation, and we state a list of propositions and lemmas, without proof, that enable us to prove Theorem 2.3. The proofs of these admitted propositions and lemmas are fully given in Section 4 and Section 5. Finally, Section 6 gives the proof of Theorem 2.1, and Section 7 collects some final comments.

\section{Surfaces, maps, and the main results}

\subsection{Surfaces, maps.}

In this paper, a surface is a connected, compact, oriented 2-manifold without boundary, considered up to oriented homeomorphism. For each integer $g \geqslant 0$, we let $\mathcal{S}_{g}$ be the $g$ torus, that is obtained from the 2 -sphere $\mathcal{S}_{0}$ by adding $g$ handles. Hence, $\mathcal{S}_{1}$ is the torus, $\mathcal{S}_{2}$ is the double torus, etc. By the theorem of classification, each surface is homeomorphic to one of the surfaces $\mathcal{S}_{g}$ for some $g \geqslant 0$ called its genus.

A map is a graph $G$ (with loops and multiple edges allowed) properly embedded into a surface $\mathbb{S}$, in such a way that the connected components of $\mathbb{S} \backslash G$, called faces, are topological disks. The genus of a map is the genus of the underlying surface. A map is bipartite if vertices of its underlying graph are coloured in black and white such that there is no monochromatic edge. Note that a bipartite map may have multiple edges but no loops. A map is rooted if an edge (called the root edge) is distinguished and oriented. The origin of the root edge is the root vertex, and the face incident to the right of the (oriented) root edge is the root face. By convention the root vertex of a bipartite map is always coloured white. We consider rooted maps up to oriented homeomorphisms preserving the root edge and its orientation. The degree of a vertex in a bipartite map is its degree in the underlying multigraph, i.e. the number of edges incident to it, with multiplicity. The degree of a face in a bipartite map is the number of edges bounding this 


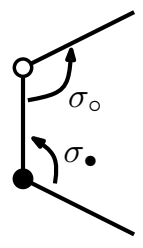

(a)

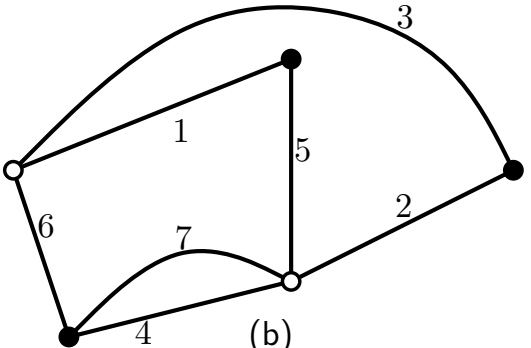

(b)

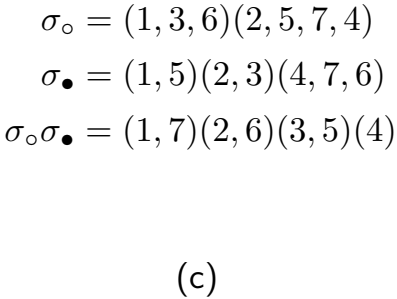

(c)

Figure 1: A labelled bipartite map with 7 edges in the plane, and the corresponding permutations $\sigma_{\circ}, \sigma_{\bullet}, \sigma_{\circ} \sigma_{\bullet}$.

face, counted with multiplicity. Because colours alternate along an edge, the degree of faces in a bipartite map are all even numbers ${ }^{1}$. If a bipartite map has $n$ edges, the sum of all face-degrees is equal to $2 n$, and the sum of all vertex-degrees of each given colour is equal to $n$.

From the algebraic viewpoint (and for the comparison with Hurwitz numbers as defined in $[18,16])$, it is sometimes convenient to consider a variant of rooted maps called labelled maps. A labelled bipartite map of size $n$ is a bipartite map with $n$ edges equipped with a labelling of its edges from 1 to $n$ such that its root edge receives label 1 . There is a 1-to- $(n-1)$ ! correspondence between rooted bipartite maps and labelled bipartite maps of size $n$. Given a labelled bipartite map, one can define two permutations $\sigma_{\circ}$ and $\sigma$ • in $\mathfrak{S}_{n}$ whose cycles record the counter-clockwise ordering of edges around white and black vertices, respectively. See Figure 1. This is a bijection between labelled bipartite maps of size $n$ and pairs $\left(\sigma_{\circ}, \sigma_{\bullet}\right)$ of permutations in $\mathfrak{S}_{n}$ such that the subgroup $\left\langle\sigma_{\circ}, \sigma_{\bullet}\right\rangle \subset \mathfrak{S}_{n}$ acts transitively on $[1 \ldots n]$. In this correspondence, cycles of $\sigma_{\circ}, \sigma_{\bullet}$, and $\sigma_{\circ} \sigma_{\bullet}$ are in natural correspondence with white vertices, black vertices, and faces, and the lengths of these cycles correspond to degrees (for vertices) and half-degrees (for faces). The genus $g$ of the underlying surface is related to the number of cycles of the three permutations $\sigma_{\circ}, \sigma_{\bullet}$ and $\sigma_{\circ} \sigma_{\bullet}$ by Euler's formula:

$$
\ell\left(\sigma_{\circ}\right)+\ell\left(\sigma_{\bullet}\right)+\ell\left(\sigma_{\circ} \sigma_{\bullet}\right)=n+2-2 g .
$$

\subsection{Notation for series and changes of variables}

In this paper, $t, x$, and $p_{1}, p_{2}, \ldots$ are indeterminates. Indices of the variables $\left(p_{i}\right)_{i \geqslant 1}$ are extended multiplicatively from integers to integer partitions, for example $p_{3,3,1}=p_{1}\left(p_{3}\right)^{2}$, and the same convention is used for other indexed sequences of variables in the paper, such as $\left(\eta_{i}\right)_{i \geqslant 1}$ or $\left(\zeta_{i}\right)_{i \geqslant 1}$.

If $\mathbb{B}$ is a ring (or field) and $s$ an indeterminate, we denote by $\mathbb{B}[s], \mathbb{B}(s), \mathbb{B}[[s]], \mathbb{B}((s)$ ), $\mathbb{B}\left(\left(s^{*}\right)\right)$ the ring (or field) of polynomials, rational functions, formal power series (f.p.s.), formal Laurent series, and Puiseux series in $s$ with coefficients in $\mathbb{B}$, respectively. If $\mathbb{B}$ is a

\footnotetext{
${ }^{1}$ Note, however that the converse is true only for genus 0 : for each genus $g \geqslant 1$, there exist maps with all faces of even degree but not bipartite (in genus 1, and example is the $m \times n$ square grid with toroidal identifications, when $m$ or $n$ is odd).
} 
field, $\overline{\mathbb{B}}$ is its algebraic closure. We will often omit the dependency of generating functions on the variables in the notation, for example we will write $L_{g}$ for $L_{g}\left(t ; p_{1}, p_{2}, \ldots\right)$ and $F_{g}$ for $F_{g}\left(t ; x ; p_{1}, p_{2}, \ldots\right)$. In this paper all fields have characteristic 0 .

Finally, an important role will be played by the "change of variables" $(t, x) \leftrightarrow(z, u)$ given by the equations:

$$
\begin{gathered}
z=t\left(1+\sum_{k \geqslant 1}\left(\begin{array}{c}
2 k-1 \\
k
\end{array}\right) p_{k} z^{k}\right), \\
u=x(1+z u)^{2} .
\end{gathered}
$$

These equations define two unique f.p.s. $z \equiv z(t) \in \mathbb{Q}\left[p_{1}, p_{2}, \ldots\right][[t]]$ and $u \equiv u(t, x) \in$ $\mathbb{Q}\left[x, p_{1}, p_{2}, \ldots\right][[t]]$. Here Equation (2) is in fact the equation for mobiles, c.f. [7]. Moreover, this change of variables is reversible, via $t(z)=\frac{z}{1+\sum_{k}\left(\begin{array}{c}2 k-1 \\ k\end{array}\right) p_{k} z^{k}}$ and $x(z, u)=\frac{u}{(1+z u)^{2}}$. Note also that, if $H \equiv H(t, x) \in \mathbb{B}[x][[t]]$ is an f.p.s. in $t$ with polynomial coefficients in $x$ over some ring $\mathbb{B}$ containing all $p_{i}$, then $H(t(z), x(z, u))$ is an element of $\mathbb{B}[u][[z]]$. In this paper we are going to abuse notation and we will switch without warning between a series $H \in \mathbb{B}[x][[t]]$ and its image in $\mathbb{B}[u][[z]]$ via the change of variables. We are going to use the single letter $H$ for both objects, relying on the context that should prevent any misunderstanding.

\subsection{Generating functions and the main result}

For $n \geqslant 1$ and $\mu$ a partition of $n$ (denoted as $\mu \vdash n$ ), let $\mathfrak{l}_{g}(\mu)$ be the number of labelled bipartite maps of size $n$ and genus $g \geqslant 0$ whose half-face degrees are given by the parts of $\mu$. Equivalently:

$$
\mathfrak{l}_{g}(\mu):=\#\left\{\begin{array}{c}
\left(\sigma_{\circ}, \sigma_{\bullet}\right) \in\left(\mathfrak{S}_{n}\right)^{2} ; \quad \ell\left(\sigma_{\circ}\right)+\ell\left(\sigma_{\bullet}\right)+\ell\left(\sigma_{\circ} \sigma_{\bullet}\right)=n+2-2 g ; \\
\left\langle\sigma_{\circ}, \sigma_{\bullet}\right\rangle \text { is transitive } ; \sigma_{\circ} \sigma_{\bullet} \text { has cycle type } \mu .
\end{array}\right\} .
$$

We now form the exponential generating function of these numbers, where $t$ marks the number of edges and for $i \geqslant 1$, the variable $p_{i}$ marks the number of faces of degree $2 i$ :

$$
L_{g} \equiv L_{g}\left(t ; p_{1}, p_{2}, \ldots\right):=\mathbf{1}_{g=0}+\sum_{n \geqslant 1} \frac{t^{n}}{n !} \sum_{\mu \vdash n} \mathfrak{l}_{g}(\mu) p_{\mu},
$$

where the indicator function accounts for the unique map of genus 0 with 1 vertex and 0 edge, that we allow by convention. Similarly, for $n, k \geqslant 1$ and $\mu \vdash n-k$, we let $\mathfrak{b}_{g}(k ; \mu)$ be the number of rooted bipartite maps of genus $g$ with $n$ edges, such that the root face has half-degree $k$, and the half-degrees of non-root faces are given by the parts of $\mu$. We let $F_{g} \equiv F_{g}\left(t ; x ; p_{1}, p_{2}, \ldots\right)$ be the corresponding ordinary generating function:

$$
F_{g} \equiv F_{g}\left(t ; x ; p_{1}, p_{2}, \ldots\right):=\mathbf{1}_{g=0}+\sum_{n \geqslant 1} t^{n} \sum_{\substack{k \geqslant 1 \\ \mu \vdash n-k}} \mathfrak{b}_{g}(k, \mu) x^{k} p_{\mu} .
$$

Our first main result is the following theorem: 
Theorem 2.1 (Main result - labelled unrooted case $(\boldsymbol{g} \geqslant \mathbf{2})$ ). Let $z \equiv z(t)$ be the unique formal power series defined by (2). Moreover, define the "variables" $\eta$ and $\zeta$ as the following formal power series:

$$
\eta=\sum_{k \geqslant 1}(k-1)\left(\begin{array}{c}
2 k-1 \\
k
\end{array}\right) p_{k} z^{k}, \quad \zeta=\sum_{k \geqslant 1} \frac{k-1}{2 k-1}\left(\begin{array}{c}
2 k-1 \\
k
\end{array}\right) p_{k} z^{k}
$$

and the variables $\left(\eta_{i}\right)_{i \geqslant 1}$ and $\left(\zeta_{i}\right)_{i \geqslant 1}$ by

$\eta_{i}:=\sum_{k \geqslant 1}(k-1) k^{i}\left(\begin{array}{c}2 k-1 \\ k\end{array}\right) p_{k} z^{k}, \quad \zeta_{i}=\sum_{k \geqslant 1} \frac{(-2)^{i+1} k(k-1) \cdots(k-i)}{(2 k-1)(2 k-3) \cdots(2 k-2 i-1)}\left(\begin{array}{c}2 k-1 \\ k\end{array}\right) p_{k} z^{k}$.

Then for $g \geqslant 2$, the exponential generating function $L_{g}$ of labelled bipartite maps of genus $g$ is given by a finite sum:

$$
L_{g}=\sum_{\alpha, \beta, a, b} c_{a, b}^{\alpha, \beta} \frac{\eta_{\alpha} \zeta_{\beta}}{(1-\eta)^{a}(1+\zeta)^{b}}
$$

for rational numbers $c_{a, b}^{\alpha, \beta}$, where the (finite) sum is taken over integer partitions $\alpha, \beta$ and non-negative integers $a, b$, such that $|\alpha|+|\beta| \leqslant 3(g-1)$ and $a+b=\ell(\alpha)+\ell(\beta)+2 g-2$.

Example 2.1 (Generating function for the labelled unrooted case, genus 2).

$$
\begin{aligned}
& L_{2}=\frac{1}{120}-\frac{1}{23040} \frac{\eta_{1}\left(185 \eta_{1}-58 \eta_{2}\right)}{(1-\eta)^{4}}-\frac{1}{46080} \frac{20 \eta_{3}-168 \eta_{2}+415 \eta_{1}}{(1-\eta)^{3}}-\frac{53 / 15360}{(1-\eta)^{2}} \\
& -\frac{7}{2880} \frac{\eta_{1}{ }^{3}}{(1-\eta)^{5}}-\frac{1 / 512}{(1-\eta)(1+\zeta)}+\frac{\eta_{1} / 1536}{(1-\eta)^{2}(1+\zeta)}-\frac{3}{1024} \frac{1}{(1+\zeta)^{2}}+\frac{3}{8192} \frac{\zeta_{1}}{(1+\zeta)^{3}} .
\end{aligned}
$$

The case of genus 1 is stated separately since it involves logarithms:

Theorem 2.2 (Labelled unrooted case, genus 1). The exponential generating function $L_{1} \equiv L_{1}\left(t ; p_{1}, p_{2}, \ldots\right)$ of bipartite maps on the torus is given by the following expression, with the notation of Theorem 2.1:

$$
L_{1}=\frac{1}{24} \ln \frac{1}{1-\eta}+\frac{1}{8} \ln \frac{1}{1+\zeta} .
$$

In order to establish Theorem 2.1 we will first prove its (slightly weaker) rooted counterpart:

Theorem 2.3 (Main result - rooted case). Let $z \equiv z(t)$ and $u=u(x, t)$ be defined by $(2)-$ (3), and let the variables $\eta, \zeta$ and $\left(\eta_{i}\right)_{i \geqslant 1}$ and $\left(\zeta_{i}\right)_{i \geqslant 1}$ be defined as in Theorem 2.1. Then for all $g \geqslant 1$, the generating function $F_{g} \equiv F_{g}\left(t ; x ; p_{1}, p_{2}, \ldots\right)$ of rooted bipartite maps of genus $g$ is equal to

$$
F_{g}=\sum_{c=1}^{6 g-1} \sum_{\alpha, \beta, a \geqslant 0, b \geqslant 0} \frac{\eta_{\alpha} \zeta_{\beta}}{(1-\eta)^{a}(1+\zeta)^{b}}\left(\frac{d_{a, b, c,+}^{\alpha, \beta}}{(1-u z)^{c}}+\frac{d_{a, b, c,-}^{\alpha, \beta}}{(1+u z)^{c}}\right),
$$


for $d_{a, b, c, \pm}^{\alpha, \beta} \in \mathbb{Q}$, with the same notation as in Theorem 2.1. Furthermore, $d_{a, b, c, \pm}^{\alpha, \beta} \neq 0$ implies $(2 \pm 1) g \geqslant\left\lceil\frac{1+c}{2}\right\rceil+|\alpha|+|\beta|$ and $a+b=\ell(\alpha)+\ell(\beta)+2 g-1$ for the two signs, and the sum above is finite.

\section{Some comments on the theorems.}

- Note that the "Greek" variables $\eta, \zeta, \eta_{i}, \zeta_{i}$ are all infinite linear combinations of the $p_{k} z^{k}$ with explicit coefficients. Moreover, for fixed $g$ the sums (5), (4) depend only of finitely many Greek variables, see e.g. Example 2.1. Note also that if only finitely many $p_{i}$ 's are nonzero, then all the Greek letters are polynomials depending unique on variable $z$. For example, if $p_{i}=\mathbf{1}_{i=2}$, i.e. if we enumerate bipartite quadrangulations, all Greek variables are polynomials in the variable $s(=z+1)$ defined in Equation (1). In particular, and since bipartite quadrangulations are in bijection with general rooted maps (see e.g. [21]), the rationality results of [2] are a (very) special case of our results.

- Readers familiar with the bijective techniques of map enumeration will notice that the change of variables $(t, x) \leftrightarrow(z, u)$ is very natural in view of the link between bipartite maps and mobiles $[7,9]$. However, those bijections are still far from giving combinatorial proofs of Theorems 2.3 and 2.1 .

- The case of genus 0 is not covered by the theorems above but is well known, and we will use it thoroughly. See Proposition 3.2 below.

We conclude this section with a last notation that will be useful throughout the paper. In addition to the "Greek" variables $\eta, \zeta,\left(\eta_{i}\right)_{i \geqslant 1},\left(\zeta_{i}\right)_{i \geqslant 1}$ already defined, we introduce the variable $\gamma$ as the following formal power series:

$$
\gamma:=\sum_{k \geqslant 1}\left(\begin{array}{c}
2 k-1 \\
k
\end{array}\right) p_{k} z^{k}
$$

Note that the change of variables (2) relating $z$ to $t$ is given by $z=t(1+\gamma)$.

\section{The Tutte equation, and the outline of the proof of Theo- rem 2.3}

\subsection{The Tutte/Lehman-Walsh equation}

In this section, we state the equation that is the starting point of our proofs. We first define some useful operators. The rooting operator $\Gamma$ is defined by

$$
\Gamma:=\sum_{k \geqslant 1} k x^{k} \frac{\partial}{\partial p_{k}} .
$$

Combinatorially, the effect of $\Gamma$ is to mark a face of degree $2 k$, distinguish one of its $k$ white corners, and record the size of this face using the variable $x$. In other words, $\Gamma$ is the operator that selects a root face in a map. From the discussion of Section 2.1, it is easy to see that $F_{g}=\Gamma L_{g}$. Note that the operator $\Gamma$ behaves as a differentiation, i.e. it satisfies $\Gamma(A B)=A \Gamma B+B \Gamma A$. 
If $F \equiv F(x)$ is an f.p.s whose coefficients are polynomials in $x$ (over some ring), we let $\Delta F(x)=\frac{F(x)-F(0)}{x}$. Equivalently, $\Delta F(x)=\left[x^{\geqslant 0}\right] \frac{1}{x} F(x)$ where $\left[x^{\geqslant 0}\right]$ is the operator that selects monomials with non-negative powers in $x$. We define the operator:

$$
\Omega:=\sum_{k \geqslant 1} p_{k} \Delta^{k}=\left[x^{\geqslant 0}\right] \sum_{k \geqslant 1} \frac{p_{k}}{x^{k}} .
$$

Proposition 3.1 (Tutte/Lehman-Walsh/loop equation - classical). The sequence $\left(F_{g}\right)_{g \geqslant 0}$ of formal power series in $\mathbb{Q}\left[p_{1}, p_{2}, \ldots\right][x][[t]]$ is uniquely determined by the equations, for $g \geqslant 0$ :

$$
F_{g}=\mathbf{1}_{g=0}+x t \Omega F_{g}+x t F_{g-1}^{(2)}+x t \sum_{\substack{g_{1}+g_{2}=g \\ g_{1}, g_{2} \geqslant 0}} F_{g_{1}} F_{g_{2}},
$$

where $F_{g-1}^{(2)}:=\Gamma F_{g-1}$ is the g.f. of bipartite maps of genus $g$ with two root faces.

Figure 2: A, B, C: Decomposition of a bipartite map by removing the root edge, leading to the three terms of the Tutte equation (8). The root face is represented in (green) rising hatching pattern, and the root edge is in (blue) fat width. TopRight: The operator that
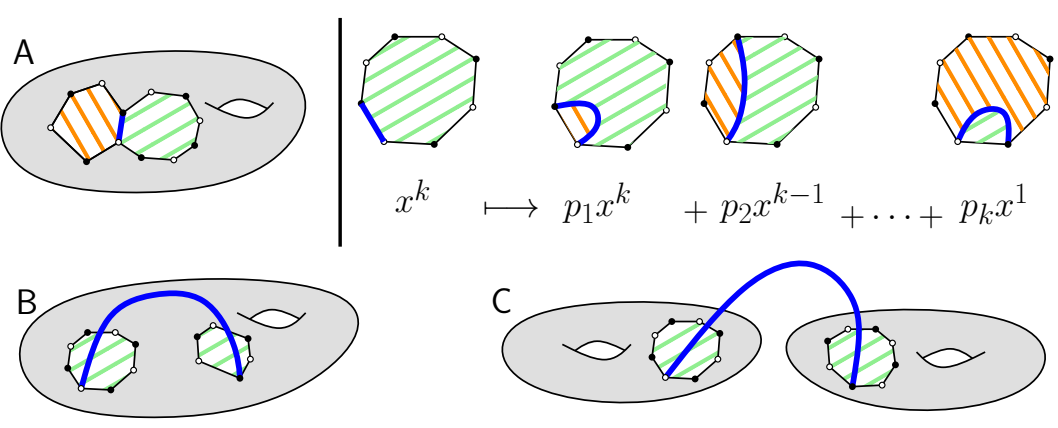
handles case A on generating functions.

Proof. This equation and its variants for different families of maps are classical and go back at least to Lehman and Walsh [23] who wrote the analogue for non-bipartite maps. The planar case $(g=0)$ goes back to Tutte who wrote and used such equations extensively in his "Census" series (e.g. [22]), hence the generic terminology "Tutte equations". They are also well known to physicists and called "loop equations" in different contexts (see again $[20,14]$ and references therein). Therefore, we will just briefly recall where the different terms come from in the present case. Start with a rooted bipartite map $\mathfrak{m}$ with at least one edge (the case of the empty map with 0 edge is taken into account by the indicator function in (8)). We distinguish three cases: A: Removing $e$ does not disconnect $\mathfrak{m}$, and $e$ is bordered by two different faces in $\mathfrak{m}$; B. Removing $e$ does not disconnect $\mathfrak{m}$, and $e$ is bordered twice by the same face in $\mathfrak{m}$; C. Removing $e$ disconnects $\mathfrak{m}$. Using Euler's formula it is easy to see that in cases A,B,C, the map $\mathfrak{m} \backslash e$ has genus $g$, genus $g-1$, and two connected components of genera adding up to $g$, respectively. We leave the reader to check (with the help of Figure 2) that the three last terms in (8) account for these three cases. 
In genus 0, the Tutte equation (8) was solved by Bender and Canfield [3] who gave the following remarkable expression in terms of the variables $z \equiv z(t), u \equiv u(t ; x)$ defined by $(2)-(3)$ :

Proposition 3.2 (Bender and Canfield [3]). The generating function of rooted bipartite maps of genus 0 is given by:

$$
F_{0}=(1+u z)\left(1-\sum_{k=1}^{K} p_{k} z^{k} \sum_{\ell=1}^{k-1} u^{\ell} z^{\ell}\left(\begin{array}{c}
2 k-1 \\
k+\ell
\end{array}\right)\right) .
$$

The strategy we will use to prove Theorem 2.3 is to solve (8) recursively on the genus $g$. Note that, for $g \geqslant 1$, and assuming that all the series $F_{h}, F_{h}^{(2)}$ are known for $h<g$, the Tutte equation (8) is linear in the unknown series $F_{g}(x)$. More precisely it is a linear "catalytic" equation for the unknown series $F_{g}$ involving one catalytic variable (the variable $x$ ), see e.g. [5]. Therefore, it is tempting to solve it via the kernel method or one of its variants.

In what follows, in order to make the induction step feasible, we will need to fix an arbitrary integer $K \geqslant 2$, and to make the substitution $p_{i}=0$ for $i>K$ in (8). The integer $K$ will be sent to infinity at the end of the induction step. To prevent a possible misunderstanding, we warn the reader that the substitution of $p_{i}$ to zero does not commute with $\Gamma$, and in particular:

$$
\left.F_{g}^{(2)}\right|_{p_{i}=0}=\left.\left(\Gamma F_{g}\right)\right|_{p_{i}=0} \neq \Gamma\left(\left.F_{g}\right|_{p_{i}=0}\right) .
$$

In concrete terms, even after we set the variables $p_{i}$ to zero for all $i>K$, the series $F_{g}^{(2)}$ still counts maps in which the two root faces may have arbitrarily large degrees. We now proceed with the inductive part of the proof, that will occupy the rest of this section. The base case $g=1$ of the induction will be proved here as well (with empty induction hypothesis). To formulate our induction hypothesis, we need the following notion: if $A(u)$ is a rational function over some field containing $z$, we say that $A$ is uz-symmetric if $A\left(\frac{1}{z^{2} u}\right)=A(u)$, and uz-antisymmetric if $A\left(\frac{1}{z^{2} u}\right)=-A(u)$.

Induction Hypothesis: In the rest of Section 3, we fix $g \geqslant 1$. We assume that for all genera $g^{\prime} \in[1 . . g-1]$, Theorem 2.3 holds for genus $g^{\prime}$. In particular $F_{g^{\prime}}$ is a rational function of $u$. We assume that it is uz-antisymmetric.

We now start examining the induction step. From now on, we assume that $\boldsymbol{p}_{\boldsymbol{i}}=\mathbf{0}$ for $\boldsymbol{i}>\boldsymbol{K}$. In other words, each series mentioned below is considered under the substitution $\left\{p_{i}=0, i>K\right\}$, even if the notation does not make it apparent. Our first observation is the following:

Proposition 3.3 (Kernel form of the Tutte equation). Define $Y:=1-2 t x F_{0}-t x \theta$, where $\theta:=\sum_{k=1}^{K} \frac{p_{k}}{x^{k}}$. Then one has:

$$
Y F_{g}=x t F_{g-1}^{(2)}+x t \sum_{\substack{g_{1}+g_{2}=g \\ g_{1}, g_{2}>0}} F_{g_{1}} F_{g_{2}}+x t S
$$


where $S \equiv S\left(t, p_{1}, p_{2}, \ldots ; x\right)$ is an element of $\mathbb{Q}\left[p_{1}, p_{2}, \ldots\right][[t]]\left[\frac{1}{x}\right]$ of degree at most $K-1$ in $\frac{1}{x}$ without constant term.

Proof. Consider the Tutte equation (8). Keeping in mind that we have done the substitution $p_{i}=0$ for $i>K$, we observe that $\Omega F_{g}=\left[x^{\geqslant 0}\right] F_{g} \theta$. Now let $S$ be the negative part of $F_{g} \theta$, i.e:

$$
S:=\left[x^{<0}\right] F_{g} \theta=F_{g} \theta-\left[x^{\geqslant 0}\right] F_{g} \theta .
$$

Now note that $\Omega F_{g}=\theta F_{g}-S$, and substitute this relation in (8). By moving to the left all the terms involving $F_{g}$ and factoring out $F_{g}$, we obtain (10). Moreover, since $\theta$ is in $\mathbb{K}\left[x^{-1}\right]$ and has degree $K$, and since $F_{g}$ has no constant terms in $x, S$ is also in $\mathbb{K}\left[x^{-1}\right]$ and has degree at most $K-1$.

\subsection{Rational structure of $F_{g}$ and the topological recursion}

In this section we describe in detail the structure of the kernel $Y$ and of the generating function $F_{g}$, in order to establish our main recurrence equation (Theorem 3.9). We leave the proofs of the most technical statements to Section 5 and Section 4 .

In order to analyse Proposition 3.3 it is natural to study the properties of the "kernel" $Y$. In what follows, we will consider elements in $\mathbb{A}[z][u]$ or $\mathbb{A}[[z]][u]$ where $\mathbb{A}:=$ $\mathbb{Q}\left(p_{1}, p_{2}, \ldots, p_{K}\right)$. Note that any such element, viewed as a polynomial in $u$, is split over $\mathbb{P}:=\overline{\mathbb{A}}\left(\left(z^{*}\right)\right)$. An element $u_{0} \in \mathbb{P}$ is large if it starts with a negative power in $z$, and is small otherwise. The following result is a consequence of (9) and some computations that we delay to Section 5. As explained in Section 2.2, it is implicit in the following that generating functions are considered under the change of variables $(t, x) \leftrightarrow(z, u)$ :

Proposition 3.4 (Rational structure of the kernel). The kernel $Y$ is an element of $\mathbb{Q}\left(z, u, p_{1}, p_{2}, \ldots, p_{K}\right)$ of the form:

$$
Y=\frac{N(u)(1-u z)}{u^{K-1}(1+\gamma)(1+u z)}
$$

where $N(u) \in \mathbb{A}[z][u]$ is a polynomial of degree $2(K-1)$ in $u$.

Proof. See Section 5.

Proposition 3.5 (Structure of zeros of the kernel).

(1) $Y$ is uz-antisymmetric.

(2) Among the $2(K-1)$ zeros of $N(u)$ in $\mathbb{P},(K-1)$ of them are small and $(K-1)$ are large. These large and small zeros are exchanged by the transformation $u \leftrightarrow \frac{1}{z^{2} u}$.

Proof. See Section 5. 
Before solving (10), we still need to examine more closely the structure of $F_{g}$. In what follows each rational function $R(u) \in \mathbb{B}(u)$ for some field $\mathbb{B}$ is implicitly considered as an element of $\overline{\mathbb{B}}(u)$. In particular its denominator is split, and the notion of pole is well defined (poles are elements of $\overline{\mathbb{B}}$ ). Moreover, $R(u)$ has a partial fraction expansion, with coefficients in $\overline{\mathbb{B}}$, and the residue of $R(u)$ at a pole $u_{*} \in \overline{\mathbb{B}}$ is a well defined element of $\overline{\mathbb{B}}$, namely the coefficient of $\left(u-u_{*}\right)^{-1}$ in this expansion. The following result is perhaps the most crucial conceptual step of the topological recursion and of the proof of Theorem 2.3:

Proposition 3.6 (Structure and poles of $\boldsymbol{F}_{\boldsymbol{g}}$ ). $F_{g}$ is an uz-antisymmetric element of $\mathbb{A}[[z]](u)$. Its poles, that are elements of $\mathbb{P}$, are contained in $\left\{\frac{1}{z},-\frac{1}{z}\right\}$. Moreover, $F_{g}$ has negative degree in $u$.

The proof of Proposition 3.6 uses the next two lemmas:

Lemma 3.7. If $A$ is an element of $\mathbb{Q}\left(u, z, \gamma, \eta, \zeta,\left(\eta_{i}\right)_{i \geqslant 1},\left(\zeta_{i}\right)_{i \geqslant 1}\right)$ with negative degree in $u$ whose poles in $u$ are among $\left\{ \pm \frac{1}{z}\right\}$, then so is $\Gamma A(u)$. Moreover, if $A(u)$ is uzantisymmetric, then $\Gamma A(x)$ is uz-symmetric.

Proof. See Section 4.2.

Lemma 3.8. Let $A(u) \in \mathbb{B}[[z]](u) \cap \mathbb{B}[u]((z)) \subset \mathbb{B}(u)((z))$. Then, when seen as a rational function in $u, A(u)$ has no small pole.

Proof. By the Newton-Puiseux theorem, we can write $A(u)=\frac{P(u)}{c \cdot Q_{1}(u) Q_{2}(u)}$ with $P(u) \in$ $\mathbb{B}[[z]][u], c \in \overline{\mathbb{B}}\left(\left(z^{*}\right)\right), Q_{1}(u)=\prod_{i}\left(1-u u_{i}\right)$ and $Q_{2}(u)=\prod_{j}\left(u-v_{j}\right)$, where the $u_{i}, v_{j}$ are small Puiseux series over an algebraic closure $\overline{\mathbb{B}}$ of $\mathbb{B}$ and $v_{j}$ without constant term. Since $P(u) / Q_{2}(u)=c A(u) Q_{1}(u)$, and since $\overline{\mathbb{B}}[u]\left(\left(z^{*}\right)\right)$ is a ring, we see that $P(u) / Q_{2}(u) \in$ $\overline{\mathbb{B}}[u]\left(\left(z^{*}\right)\right)$. But since $1 / Q_{2}(u)=\prod_{j} \sum_{k \geqslant 0} u^{-1-k} v_{i}^{k}$ is in $\overline{\mathbb{B}}\left[u^{-1}\right]\left(\left(z^{*}\right)\right)$, this is impossible unless $Q_{2}$ divides $P$ in $\overline{\mathbb{B}}\left(\left(z^{*}\right)\right)[u]$, which concludes the proof.

We can now give the proof of Proposition 3.6:

Proof of Proposition 3.6. We first claim that the right-hand side of (10) is uz-symmetric. In the case $g \geqslant 2$ this follows by induction, since each term $F_{g_{1}} F_{g_{2}}$ is $u z$-symmetric as a product of two $u z$-antisymmetric factors, the term $F_{g-1}^{(2)}$ is $u z$-symmetric using Lemma 3.7, and $S$, as any rational fraction in $x$, is symmetric since $x(u)=\frac{u}{(1+z u)^{2}}$ is symmetric. In the case $g=1$, the R.H.S. of $(10)$ is equal to $x t F_{0}^{(2)}+x t S$, so it is enough to see that $F_{0}^{(2)}$ is $u z$-symmetric. Now, the series $F_{0}^{(2)}$ is given by the explicit expression:

$$
F_{0}^{(2)}=\frac{u^{2} z^{2}}{(1-u z)^{4}}
$$

This expression can be found in [13] (recall that what [13] calls bipartite maps do not coincide with bipartite maps in general, but they coincide in genus 0 , so we can use this result here). It can also be obtained from direct computations from the explicit expression 
of $F_{0}$ given by Proposition 3.2, and it is also easily derived from [11, Thm. 1] (in the case $p=r=2$, with the notation of that reference). Since (11) is clearly $u z$-symmetric, the claim is proved in all cases.

Hence, by Proposition $3.5, F_{g}$ is $u z$-antisymmetric, being the quotient of the $u z$ symmetric right-hand side of (10) by $Y$. Now, by the induction hypothesis and Lemma 3.7 (or by a direct check on (11) in the case $g=1$ ), the R.H.S. of $(10)$ is in $\mathbb{A}[[z]](u)$, and its poles are contained in $\left\{ \pm \frac{1}{z}, 0\right\}$. Hence, by solving (10) for $F_{g}$ and by using Proposition 3.4, we deduce that $F_{g}$ belongs to $\mathbb{A}[[z]](u)$ and that its only possible poles are $\pm \frac{1}{z}, 0$ and the zeros of $N(u)$.

Now, viewed as a series in $z, F_{g}$ is an element of $\mathbb{A}[u][[z]]$. Indeed, in the variables $(t, x), F_{g}$ belongs to $\mathbb{Q}\left[p_{1}, \ldots, p_{K}\right][x][[t]]$ for clear combinatorial reasons, and as explained in Section 2.2 the change of variables $t, x \leftrightarrow z, u$ preserves the polynomiality of coefficients. Therefore, by Lemma 3.8, $F_{g}$ has no small poles. This excludes 0 and all small zeros of $N(u)$. Since $F_{g}$ is $u z$-antisymmetric and since by Proposition 3.5, the transformation $z \leftrightarrow \frac{1}{z^{2} u}$ exchanges small and large zeros of $N(u)$, this also implies that $F_{g}$ has no pole at the large zeros of $N(u)$.

The last thing to do is to examine the degree of $F_{g}$ in $u$. We know that $S$ is a polynomial in $x^{-1}$ of degree at most $K-1$, thus has degree at most $K-1$ in $u$. Therefore, by induction and Lemma 3.7 (or by a direct check on (11) in the case $g=1$ ) the degree in $u$ of the R.H.S. of (10) is at most $K-2$. Since the degree of $Y$ is $K-1$, the degree of $F_{g}$ in $u$ is at most -1 .

Remark 3.1. Analogues of the previous proposition, stated in similar contexts [13, Chap. 3] play a crucial role in Eynard's "topological recursion" framework. To understand the importance of Proposition 3.6, let us make a historical comparison. The "traditional" way of solving (10) with the kernel method would be to substitute in (10) all the small roots of $N(u)$, and use the $(K-1)$ equations thus obtained to eliminate the "unknown" polynomial $S$. Not surprisingly, this approach was historically the first one to be considered, see e.g. [15]. It leads to much weaker rationality statements than the kind of methods we use here, since the cancellations that appear between those $(K-1)$ equations are formidable and very hard to track. As we will see, Proposition 3.6 circumvents this problem by showing that we just need to study (10) at the two points $u= \pm \frac{1}{z}$ rather than at the $(K-1)$ small roots of $N$.

With Proposition 3.6, we can now apply one of the main ideas of the topological recursion, namely that the whole object $F_{g}$ can be recovered from the expansion of (10) at the critical points $u= \pm \frac{1}{z}$. In what follows, all generating functions considered are rational functions of the variable $u$ over $\mathbb{A}[[z]]$. In particular, the notation $F_{g}(u)$ is a shorthand notation for the series $F_{g}\left(t ; x ; p_{1}, \ldots, p_{K}\right)$ considered as an element of $\mathbb{A}[[z]](u)$ (or even $\left.\mathbb{Q}\left[p_{1}, p_{2}, \ldots, p_{K}\right][[z]](u)\right)$, i.e. $F_{g}(u):=F_{g}\left(t(z), x(z, u), p_{1}, p_{2}, \ldots\right)$. We let $P(u)=\frac{1-u z}{1+u z}$ (the letter $P$ is for "prefactor"). By Proposition 3.6 the rational function $P(u) F_{g}(u)$ has only poles at $u= \pm \frac{1}{z}$ and has negative degree in $u$. Therefore, if $u_{0}$ is some new 
indeterminate, we can write $P\left(u_{0}\right) F\left(u_{0}\right)$ as the sum of two residues:

$$
P\left(u_{0}\right) F\left(u_{0}\right)=\operatorname{Res}_{u= \pm \frac{1}{z}} \frac{1}{u_{0}-u} P(u) F(u) .
$$

Note that this equality only relies on the (algebraic) fact that the sum of the residues of a rational function at all poles (including $\infty$ ) is equal to zero, no complex analysis is required. Now, multiplying $(10)$ by $P(u)$, we find:

$$
P(u) F_{g}(u)=\frac{x t P(u) H_{g}(u)}{Y(u)}+\frac{x t P(u) S(x)}{Y(u)} .
$$

with $H_{g}(u)=F_{g-1}^{(2)}(u)+\sum_{\substack{g_{1}+g_{2}=g \\ g_{1}, g_{2}>0}} F_{g_{1}}(u) F_{g_{2}}(u)$. Now observe that the second term in the right-hand side has no pole at $u= \pm \frac{1}{z}$ : indeed the factor $(1-u z)$ in $Y(u)$ simplifies thanks to the prefactor $P(u)$, and $x S(x)$ is a polynomial in $\frac{1}{x}=\frac{(1+u z)^{2}}{u}$. Returning to $(12)$ we have proved:

Theorem 3.9 (A topological recursion for bipartite maps). The series $F_{g}\left(u_{0}\right)$ can be computed as:

$$
F_{g}\left(u_{0}\right)=\frac{1}{P\left(u_{0}\right)} \operatorname{Res}_{u= \pm \frac{1}{z}} \frac{P(u)}{u_{0}-u} \frac{x t}{Y(u)}\left(F_{g-1}^{(2)}(u)+\sum_{\substack{g_{1}+g_{2}=g \\ g_{1}, g_{2}>0}} F_{g_{1}}(u) F_{g_{2}}(u)\right) .
$$

Note that the R.H.S. of (13) involves only series $F_{h}$ for $h<g$ and the series $F_{g-1}^{(2)}$, which are covered by the induction hypothesis. This contrasts with (10), where the term $S(x)$ involves small coefficients of $F_{g}$.

\subsection{Proof of Theorem 2.3.}

In order to compute $F_{g}\left(u_{0}\right)$ from Theorem 3.9, it is sufficient to be able to compute the expansion of the rational fraction $\frac{H_{g}(u)}{Y(u)}$ at the points $u= \pm \frac{1}{z}$. The expansion of the product terms $F_{g_{1}}(u) F_{g_{2}}(u)$ is well covered by the induction hypothesis, so the main point will be to study the structure of the term $F_{g-1}^{(2)}(u)$, and the derivatives of $Y(u)$ at $u= \pm \frac{1}{z}$. The first point will require close study of the action of the operator $\Gamma$ on Greek variables, and the second one requires a specific algebraic treatment. Note also that, in order to close the induction step, we will need to take the projective limit $K \rightarrow \infty$. Therefore, we need to prove not only that the derivatives of $\frac{H_{g}(u)}{Y(u)}$ at $u= \pm \frac{1}{z}$ are rational functions in the Greek variables, but also that these functions do not depend on $K$.

In the rest of this section, we apply this program and prove Theorem 2.3, using two intermediate results (Proposition 3.10 and 3.11 below), whose proofs are reported to Section 5 and 4.

The derivatives of $Y(u)$ at the critical points can be studied by explicit computations, which require some algebraic work. This is the place where we see the Greek variables appear. In Section 5.2 we will prove: 
Proposition 3.10 (expansion of $\boldsymbol{x t P}(\boldsymbol{u}) / \boldsymbol{Y}(\boldsymbol{u})$ at $\boldsymbol{u}= \pm \frac{\mathbf{1}}{\boldsymbol{z}}$ ). The rational function in $u, \frac{x t P(u)}{Y(u)}$, has the following formal expansions at $u= \pm \frac{1}{z}$ :

$$
\begin{aligned}
& \frac{x t P(u)}{Y(u)}=\frac{1}{4(1-\eta)}+\sum_{\alpha, a \geqslant 2|\alpha|} c_{\alpha, a}^{\prime \prime \prime} \frac{\eta_{\alpha}}{(1-\eta)^{\ell(\alpha)+1}}(1-u z)^{a}, \\
& \frac{x t P(u)}{Y(u)}=-\frac{1}{(1+\zeta)(1+u z)^{2}}+\sum_{\alpha, a \geqslant 2|\alpha|} c_{\alpha, a}^{\prime \prime} \frac{\zeta_{\alpha}}{(1+\zeta)^{\ell(\alpha)+1}}(1+u z)^{a-2},
\end{aligned}
$$

where $c_{\alpha, a}^{\prime \prime}, c_{\alpha, a}^{\prime \prime \prime}$ are computable rational numbers independent of $K$.

Note that the theorem above is just a formal way of collecting all the derivatives of $\frac{x t P(u)}{Y(u)}$ at $u= \pm \frac{1}{z}$, we are not interested in convergence at all here.

Before proceeding to the full proof of Theorem 2.3, we need a last proposition that details the action of the operator $\Gamma$ on Greek variables. We first introduce two notions of degrees. We let $\mathbb{G}$ be the subring of $\mathbb{Q}\left(\eta, \zeta,\left(\eta_{i}\right)_{i \geqslant 1},\left(\zeta_{i}\right)_{i \geqslant 1}, u z\right)$ formed by polynomials in the variables $(1-\eta)^{-1},(1+\zeta)^{-1},\left(\eta_{i}\right)_{i \geqslant 1},\left(\zeta_{i}\right)_{i \geqslant 1},(1-u z)^{-1},(1+u z)^{-1}$. The Greek degree and the pole degrees are defined for elements of $\mathbb{G}$. The degree of a polynomial is defined as the highest degree of a monomial with nonzero coefficient, while the degree of a monomial is defined as the product of the degrees of its factors as follows. The Greek degree, denoted by $\operatorname{deg}_{\gamma}$, depends only on Greek variables, i.e. $\operatorname{deg}_{\gamma}(s)=0$, and is defined as follows:

$$
\operatorname{deg}_{\gamma}(1-\eta)=\operatorname{deg}_{\gamma}(1+\zeta)=\operatorname{deg}_{\gamma}\left(\eta_{i}\right)=\operatorname{deg}_{\gamma}\left(\zeta_{i}\right)=1 \text { for } i \geqslant 1 .
$$

The pole degrees are defined for each of the two poles $u= \pm 1 / z$, and are denoted by $\operatorname{deg}_{+}$ and deg_. They depend on both Greek variables and $(1 \pm u z)$ as follows.

$$
\begin{aligned}
& \operatorname{deg}_{+}\left((1-u z)^{-1}\right)=1, \operatorname{deg}_{+}\left(\eta_{i}\right)=\operatorname{deg}_{+}\left(\zeta_{i}\right)=2 i \text { for } i \geqslant 1, \\
& \operatorname{deg}_{-}\left((1+u z)^{-1}\right)=1, \operatorname{deg}_{-}\left(\eta_{i}\right)=\operatorname{deg}_{-}\left(\zeta_{i}\right)=2 i \text { for } i \geqslant 1 .
\end{aligned}
$$

Proposition 3.11. If $\xi \in\left\{\zeta ; \gamma ; \eta_{i}, i \geqslant 1 ; \zeta_{i}, i \geqslant 1\right\}$ is a Greek variable, then $\Gamma \xi$ is an element of $\mathbb{G}$.

Moreover, let $T \in \mathbb{G}$ be a monomial in $(1+\zeta)^{-1},(1-\eta)^{-1},(1+u z)^{-1},(1-u z)^{-1}, \eta_{i}$ and $\zeta_{i}$ for $i \geqslant 1$. Then $\Gamma T$ is also in $\mathbb{G}$, and it is a sum of terms that are homogeneous in Greek degree. Furthermore,

$$
\operatorname{deg}_{\gamma}(\Gamma T)=\operatorname{deg}_{\gamma}(T)-1, \operatorname{deg}_{+}(\Gamma T) \leqslant \operatorname{deg}_{+}(T)+5, \operatorname{deg}_{-}(\Gamma T) \leqslant \operatorname{deg}_{-}(T)+1 .
$$

We can now prove our first main result (up to the proofs that have been omitted in what precedes, and that will be addressed in the next sections).

Proof of Theorem 2.3. We prove the theorem by induction on the genus $g \geqslant 1$.

We consider (13) in Theorem 3.9. Proposition 3.10 implies that all terms in the expansion of $x t P(u) / Y(u)$ at $u= \pm z^{-1}$, are rational fractions in the Greek variables, 
with denominator of the form $(1-\eta)^{a}(1+\zeta)^{b}$ for $a, b, \geqslant 0$. Moreover, these terms do not depend on $K$ (when written in the Greek variables). When $g \geqslant 2$, from the induction hypothesis and Proposition 3.11, the quantity $H_{g}$ is a rational fraction in $u, z$ and the Greek variables, with denominator of the form $(1-\eta)^{a}(1+\zeta)^{b}(1 \pm u z)^{c}$ for $a, b, c \geqslant 0$. This rational function does not depend on $K$ (when written in the Greek variables). The same is true for $g=1$ using the explicit expression of $F_{0}^{(2)}$ given by (11). Therefore, the evaluation of each residue in (13) is a rational function of Greek variables, independent of $K$, and with denominator of the form $(1-\eta)^{a}(1+\zeta)^{b}(1 \pm u z)^{c}$, with $a, b, c \geqslant 0$.

In order to prove Theorem 2.3, we now need to prove that $F_{g}$ is the sum of terms whose Greek degree $\operatorname{deg}_{\gamma}$ is at most $1-2 g$, and that the pole degrees of $F_{g}$ verify $\operatorname{deg}_{+}\left(F_{g}\right) \leqslant 6 g-1$ and $\operatorname{deg}_{-}\left(F_{g}\right) \leqslant 2 g-1$. Note that from the induction hypothesis, for all $g^{\prime}$ such that $1 \leqslant g^{\prime}<g$, the series $F_{g^{\prime}}$ satisfies the degree conditions above.

We first look at $H_{g}$, in the case $g \geqslant 2$. It has two parts: the sum part, which is $\sum_{g^{\prime}=1}^{g-1} F_{g^{\prime}} F_{g-g^{\prime}}$, and the operator part, which is $\Gamma F_{g-1}$. We analyse the degree for both parts. For the sum part, it is easy to see that any term $T$ in the sum is homogeneously of Greek degree $\operatorname{deg}_{\gamma}(T)=2-2 g$, and the pole degrees verify $\operatorname{deg}_{+}(T) \leqslant 6 g-2$ and $\operatorname{deg}_{-}(T) \leqslant 2 g-2$. For the operator part, it follows from Proposition 3.11 that $\Gamma F_{g-1}$ is a sum of terms $T$ homogeneously with Greek degree $2-2 g$, and $\operatorname{deg}_{+}\left(\Gamma F_{g-1}\right) \leqslant 6 g-2$, $\operatorname{deg}_{-}\left(\Gamma F_{g-1}\right) \leqslant 2 g-2$. Therefore, the results from the sum part and the operator part agree, and thus $H_{g}$ satisfies the same conditions as its two parts. For $g=1$, the same bound holds, as one can check from the explicit expression of $H_{1}=x t F_{0}^{(2)}$ following from (11).

We now observe from Proposition 3.10 that all terms appearing in the expansion of $x t P / Y$ at $u \pm \frac{1}{z}$ are homogeneously of Greek degree -1 . Therefore, all the terms in the expansion of $x t P H_{g} / Y$ at $u= \pm \frac{1}{z}$, have Greek degree $\operatorname{deg}_{\gamma}\left(H_{g}\right)+\operatorname{deg}_{\gamma}(x t P / Y)=$ $1-2 g$. For the pole degrees, we notice from Proposition 3.10 that $\operatorname{deg}_{+}(x t P / Y) \leqslant 0$ and $\operatorname{deg}_{-}(x t P / Y) \leqslant 2$. Similar to the Greek degree, counting also the contribution from $P$, we have $\operatorname{deg}_{+}\left(F_{g}\right)=\operatorname{deg}_{+}\left(H_{g}\right)+\operatorname{deg}_{+}(x t P / Y)+1 \leqslant 6 g-1$ and $\operatorname{deg}_{-}\left(F_{g}\right)=$ $\operatorname{deg}_{-}\left(H_{g}\right)+\operatorname{deg}_{-}(x t P / Y)-1 \leqslant 2 g-1$, and we complete the induction step.

We thus have proved that, under the specialisation $p_{i}=0$ for $i>K$, the series $F_{g}$ has the form stated in Theorem 2.3. But, since the numbers $d_{a, b, c, \pm}^{\alpha, \beta}$ do not depend on $K$, we can let $K \rightarrow \infty$ in (5) and conclude that this equality holds without considering this specialisation. This concludes the proof of Theorem 2.3.

\section{Overview of omitted proofs.}

We have just proved Theorem 2.3, but we have stated several intermediate results without proof, in order (we hope) to make the global structure of the proof appear more clearly. All these intermediate results will be proved in Section 4 and 5. In order to help the reader check that we do not forget any proof(!), we list here the results stated so far without proof, and indicate where their proofs belong:

- Proposition 3.11 and Lemma 3.7, that deal with the action of the operator $\Gamma$, are proved at the end of Section 4. 
- Proposition 3.4 is proved in Section 5.1, where we also prove Proposition 3.5.

- Proposition 3.10 is proved in Section 5.2. This proof is rather long, especially because we choose to evaluate the generating functions with a combinatorial viewpoint, but essentially amounts to explicit computations using the explicit expression of the series $F_{0}$.

Therefore at the end of Section 4 and 5, the proof of Theorem 2.3 will be complete (without omissions). The two remaining statements (Theorem 2.1 and 2.2) will be deduced from Theorem 2.3 in Section 6.

\section{Structure of the Greek variables and action of the operator $\Gamma$}

In this section we establish several properties of the Greek variables defined in Section 2. In particular we will prove Proposition 3.11 and Lemma 3.7. We also fix some notation that will be used in the rest of the paper.

\subsection{Properties of the Greek variables and their $\Theta$-images}

We start by fixing some notation and defining some spaces and operators that will be used throughout the rest of the paper. First we let $\mathcal{G}:=\left\{\gamma, \eta, \zeta,\left(\eta_{i}\right)_{i \geqslant 1},\left(\zeta_{i}\right)_{i \geqslant 1}\right\}$ be the set of all Greek variables defined in Theorem 2.1. Elements of $\mathcal{G}$ are infinite linear combinations of $p_{k} z^{k}$. Acting on such objects, we first define the linear operators:

$$
\begin{aligned}
& \Theta: p_{k} z^{k} \mapsto x^{k} z^{k}, \\
& D: p_{k} z^{k} \mapsto k p_{k} z^{k} .
\end{aligned}
$$

Recall that the variable $z \equiv z\left(t ; p_{1}, p_{2}, \ldots\right)$ defined by $(2)$ is an element of $\mathbb{Q}\left[p_{1}, p_{2}, \ldots\right][[t]]$ without constant term. Therefore, each formal power series $A \in \mathbb{Q}\left[x, p_{1}, p_{2}, \ldots\right][[z]]$ is an element of $\mathbb{Q}\left[x, p_{1}, p_{2}, \ldots\right][[t]]$. Recall that, on this ring, the operator $\Gamma$ is defined by: $\Gamma=\sum_{k \geqslant 1} k x^{k} \frac{\partial}{\partial p_{k}}$, where $\frac{\partial}{\partial p_{k}}$ is the partial differentiation with respect to $p_{k}$ on $\mathbb{Q}\left[x, p_{1}, p_{2}, \ldots\right][[t]]$. We now introduce another operator $\partial_{p_{k}}$, given by the partial differentiation with respect to $p_{k}$ on $\mathbb{Q}\left[x, p_{1}, p_{2}, \ldots\right][[z]]$ omitting the dependency of $z$ in $p_{k}$. Equivalently, $\partial_{p_{k}}$ is defined on $\mathbb{Q}\left[x, p_{1}, p_{2}, \ldots\right][[z]]$ by the formula:

$$
\frac{\partial}{\partial p_{k}}=\frac{\partial z}{\partial p_{k}} \frac{\partial}{\partial z}+\partial_{p_{k}}
$$

Our first statement deals with the action of $\Theta$ on elements of $\mathcal{G}$. Here and later it will be convenient to work with the variable $s$ defined by:

$$
s:=\frac{1-u z}{1+u z}
$$


Proposition 4.1. The action of the operator $\Theta$ on elements of $\mathcal{G}$ is given by:

$$
\begin{array}{rlrl}
\Theta \gamma & =\frac{1}{2}\left(s^{-1}-1\right), & \Theta \eta & =\frac{1}{4}\left(s^{-3}-3 s^{-1}+2\right), \\
\Theta \zeta & =\frac{1}{4}\left(s+s^{-1}\right)-\frac{1}{2}, & \Theta \zeta_{i} & =\left(s^{-1}-s\right)\left(s^{2}-1\right)^{i}, i \geqslant 1, \\
\Theta \eta_{i}=\frac{1}{2^{i+2}}\left(\left(s-s^{-1}\right) \frac{\partial}{\partial s}\right)^{i}\left(s^{-3}-3 s^{-1}+2\right), i \geqslant 1 . &
\end{array}
$$

In particular, the images $\Theta(\eta+\gamma), \Theta(\zeta-\gamma), \Theta \eta_{i}, \Theta \zeta_{i}$ for $i \geqslant 1$ span the vector space $\left(s^{-1}-s\right) \mathbb{Q}\left[s^{2}, s^{-2}\right]$.

Proof. The proof is elementary but let us sketch the computations that are not totally obvious if not performed in a good way. We observe, and will use several times, that by the Lagrange inversion formula, one has $\left[x^{\ell}\right] s=-\frac{2}{\ell}\left(\begin{array}{c}2 \ell-2 \\ \ell-1\end{array}\right) z^{\ell}$ for any $\ell \geqslant 1$.

- By definition we have $\Theta \gamma=\sum_{k \geqslant 1}\left(\begin{array}{c}2 k-1 \\ k\end{array}\right) x^{k} z^{k}$, so to prove the first equality we need to show that for $k \geqslant 1$ one has $\left[x^{k}\right] \frac{1}{2} s^{-1}=\left(\begin{array}{c}2 k-1 \\ k\end{array}\right) z^{k}$. For this, we first observe by a direct computation that $s^{2}=1-4 x z$, which implies that $2 x \frac{\partial}{\partial x} s=s-s^{-1}$. It follows that $\left[x^{k}\right] \frac{1}{2} s^{-1}=\left[x^{k}\right] \frac{1}{2}\left(s-2 x \frac{\partial}{\partial x} s\right)=(1-2 k)\left[x^{k}\right] s$, which is equal to $\left(\begin{array}{c}2 k-1 \\ k\end{array}\right) z^{k}$ from the observation above. The value of $\Theta \zeta$ is easily checked similarly, namely $\left[x^{k}\right]\left(s+s^{-1}\right) / 4=$ $\left[x^{k}\right]\left(s-x \frac{\partial}{\partial x} s\right) / 2=\frac{1-k}{2}\left[x^{k}\right] s=\frac{k-1}{2 k-1}\left(\begin{array}{c}2 k-1 \\ k\end{array}\right) z^{k}$.

To check the value of $\Theta \zeta_{i}$, we observe again that $s^{2}-1=-4 x z$, so that $\left[x^{k}\right]\left(s^{-1}-s\right)\left(s^{2}-\right.$ $1)^{i}=(-4 z)^{i}\left[x^{k-i}\right]\left(s^{-1}-s\right)$. Using again that $2 x \frac{\partial}{\partial x} s=s-s^{-1}$, this is equal to $(-4 z)^{i}$. $2(i-k)\left[x^{k-i}\right] s$, which equals $(-1)^{i+1} 2^{2 i+1}\left(\begin{array}{c}2 k-2 i-2 \\ k-i-1\end{array}\right) z^{k}$. This quantity can be rewritten as $\frac{(-2)^{i+1} k(k-1) \ldots(k-i)}{(2 k-1)(2 k-3) \ldots(2 k-2 i-1)}\left(\begin{array}{c}2 k-1 \\ k\end{array}\right) z^{k}$ that agrees with what we expect from the definition of $\zeta_{i}$.

To compute $\Theta \eta$ and $\Theta \eta_{i}$, we first notice that $\Theta D=x \frac{\partial}{\partial x} \Theta$, and we observe that

$$
\eta=D \gamma-\gamma, \eta_{1}=D \eta, \eta_{i}=D \eta_{i-1} .
$$

We can then compute the action of $\Theta$ on these variables.

$$
\begin{gathered}
\Theta \eta=\left(x \frac{\partial}{\partial x}-I d\right) \Theta \gamma=\frac{1}{4}\left(s^{-3}-3 s^{-1}+2\right) \\
\Theta \eta_{i}=\left(x \frac{\partial}{\partial x}\right)^{i} \Theta \eta=\frac{1}{2^{i+2}}\left(\left(s-s^{-1}\right) \frac{\partial}{\partial s}\right)^{i}\left(s^{-3}-3 s^{-1}+2\right)
\end{gathered}
$$

- We now prove the last statement of the proposition. We have $\Theta(\zeta-\gamma)=\left(s-s^{-1}\right) / 4$ and $\Theta \zeta_{i}=\left(s^{-1}-s\right)\left(s^{2}-1\right)^{i}$ of degree $2 i+1$ in $s$, and they form a triangular basis of $\left(s^{-1}-\right.$ $s) \mathbb{Q}\left[s^{2}\right]$. We also observe that $\Theta(\eta+\gamma)=\left(s-s^{-1}\right) s^{-2} / 4$ and $\Theta \eta_{i}$ is in $\left(s^{-1}-s\right) s^{-2} \mathbb{Q}\left[s^{-2}\right]$ of degree $2 i+1$ in $s^{-1}$, and also that they form a triangular basis for $\left(s^{-1}-s\right) s^{-2} \mathbb{Q}\left[s^{-2}\right]$. This proves that altogether these variables span the whole desired space.

The next proposition, which is a simple check from the definitions using implicit differentiation, collects some partial derivatives of our main variables that will be useful afterwards. 
Proposition 4.2. The partial derivatives relating the variable sets $t, x$ and $z, u$ are given by:

$$
\begin{aligned}
\frac{\partial u}{\partial x}=\frac{(1+u z)^{3}}{1-u z}, \quad \frac{\partial z}{\partial t}=\frac{(1+\gamma)^{2}}{1-\eta}, & \frac{\partial u}{\partial t}=\frac{2(1+\gamma)^{2} u^{2}}{(1-\eta)(1-u z)} \\
\frac{\partial z}{\partial x}=0, \quad \frac{\partial z}{\partial p_{k}}=\frac{\left(\begin{array}{c}
2 k-1 \\
k
\end{array}\right) z^{k+1}}{1-\eta}, & \frac{\partial u}{\partial p_{k}}=\frac{2 u^{2}\left(\begin{array}{c}
2 k-1 \\
k
\end{array}\right) z^{k+1}}{(1-u z)(1-\eta)}
\end{aligned}
$$

\subsection{Action of $\Gamma$ and proofs of Proposition 3.11 and Lemma 3.7}

We are now ready to study more explicitly the action of $\Gamma$.

Proposition 4.3. We have

$$
\Gamma z=\frac{z s^{-2}\left(s^{-1}-s\right)}{4(1-\eta)}, \quad \Gamma u=\frac{u s^{-2}\left(s^{-1}-1\right)\left(s^{-1}-s\right)}{4(1-\eta)}, \quad \Gamma s=-\frac{\left(s^{-1}-s\right)^{2}}{8(1-\eta) s^{2}}
$$

Proof. We proceed by direct computation by recalling the differentials computed in Proposition 4.2 .

$$
\begin{aligned}
\Gamma z & =\sum_{k \geqslant 1} k x^{k} \frac{\partial}{\partial p_{k}} t\left(1+\sum_{m \geqslant 1}\left(\begin{array}{c}
2 m-1 \\
m
\end{array}\right) p_{m} z^{m}\right) \\
& =\sum_{k \geqslant 1} k\left(\begin{array}{c}
2 k-1 \\
k
\end{array}\right) x^{k} z^{k}+\frac{1}{1+\gamma}(\Gamma z) \sum_{k \geqslant 1} k\left(\begin{array}{c}
2 k-1 \\
k
\end{array}\right) p_{k} z^{k} \\
& =\frac{z}{1+\gamma} \Theta(\gamma+\eta)+\frac{1}{1+\gamma}(\Gamma z)(\gamma+\eta)
\end{aligned}
$$

By solving this linear equation, we obtain $\Gamma z$. To obtain $\Gamma u$, we notice that $\Gamma$ is a derivation and apply it to $x=u(1+u z)^{2}$ to obtain

$$
0=(\Gamma u)(1+u z)^{-3}(1-u z)-(\Gamma z) 2 u^{2}(1+u z)^{-3} .
$$

Finally, using the fact that $\Gamma$ is a derivation and the values of $\Gamma z$ and $\Gamma u$, we easily compute $\Gamma s$.

Proposition 4.4. For $G$ a linear combination of elements of $\mathcal{G}$, we have

$$
\Gamma G=\left(\frac{s^{-1}-s}{4(1-\eta) s^{2}}+\Theta\right) D G
$$

Proof. Since $G$ is a linear combination of Greek variables, it is an infinite linear combination of $p_{k} z^{k}$. Recalling the definition (16) of the operator $\partial_{p_{k}}$, we have:

$$
\begin{aligned}
\Gamma G=\sum_{k \geqslant 1} k x^{k} \frac{\partial}{\partial p_{k}} G & =\sum_{k \geqslant 1} k x^{k} \frac{\partial z}{\partial p_{k}} \frac{\partial}{\partial z} G+\sum_{k \geqslant 1} k x^{k} \partial_{p_{k}} G \\
& =\sum_{k \geqslant 1} k x^{k} \frac{\partial z}{\partial p_{k}} z^{-1} D G+\Theta D G \\
& =\left(z^{-1}(\Gamma z)+\Theta\right) D G=\left(\frac{s^{-1}-s}{4(1-\eta) s^{2}}+\Theta\right) D G .
\end{aligned}
$$


We are now prepared to prove Proposition 3.11 and Lemma 3.7.

Proof of Proposition 3.11. To obtain explicit formulas giving the action of $\Gamma$, we use Proposition 4.4. For $G \in \mathcal{G}$, the value of $D G$ is given by the following list, which is straightforward from the definitions:

$$
\begin{aligned}
& D \gamma=\eta+\gamma ; D \eta=\eta_{1} ; \quad D \zeta=\frac{\eta+\zeta}{2} ; \quad D \eta_{i}=\eta_{i+1} \\
& D \zeta_{i}=\frac{1}{2}\left((2 i+1) \zeta_{i}+\sum_{j=1}^{i-1}(-1)^{j-1} \zeta_{i-j}+4(-1)^{i}(\zeta+\eta)\right) .
\end{aligned}
$$

Since all the quantities appearing in the right-hand side of these equalities are linear combinations of elements of $\mathcal{G}$, their images by $\Theta$ can be computed thanks to Proposition 4.1. Therefore, using Proposition 4.4, we can compute explicitly the value of $\Gamma G$ for $G \in \mathcal{G}$, which leads to the following expressions:

$$
\begin{aligned}
\Gamma \zeta_{i}= & \frac{s^{-1}-s}{8(1-\eta) s^{2}}\left((2 i+1) \zeta_{i}+\sum_{j=1}^{i-1}(-1)^{j-1} \zeta_{i-j}+4(-1)^{i}(1+\zeta)\right) \\
& \quad+\frac{1}{2}\left(s^{-1}-s\right)\left((2 i+1)\left(s^{2}-1\right)^{i}+\sum_{j=1}^{i-1}(-1)^{j-1}\left(s^{2}-1\right)^{i-j}+(-1)^{i}\right) \\
\Gamma \zeta= & \frac{s^{-1}-s}{8(1-\eta) s^{2}}(\eta+\zeta)+\frac{1}{8}\left(s^{-3}-s^{-1}-2+2 s\right) \\
\Gamma \gamma= & \frac{s^{-1}-s}{4(1-\eta) s^{2}}(\eta+\gamma)+\frac{1}{4}\left(s^{-3}-s^{-1}\right) \\
\Gamma \eta_{i}= & \frac{s^{-1}-s}{4(1-\eta) s^{2}} \eta_{i+1}+\frac{1}{2^{i+3}}\left(\left(s-s^{-1}\right) \partial_{s}\right)^{i+1}\left(s^{-3}-3 s^{-1}+2\right),
\end{aligned}
$$

where $s=\frac{1-u z}{1+u z}$. This proves the first part of the proposition.

To prove the second part (concerning the degrees), we fix a monomial $T \in \mathbb{G}$ as in the statement of the proposition. Since $\Gamma$ is a derivation, we have the following expression for $\Gamma T$.

$$
\Gamma T=(\Gamma u z) \frac{\partial}{\partial(u z)} T+(\Gamma \zeta) \frac{\partial}{\partial \zeta} T+(\Gamma \eta) \frac{\partial}{\partial \eta} T+\sum_{i \geqslant 1}\left(\Gamma \eta_{i}\right) \frac{\partial}{\partial \eta_{i}} T+\sum_{i \geqslant 1}\left(\Gamma \zeta_{i}\right) \frac{\partial}{\partial \zeta_{i}} T
$$

It suffices to analyse the degree of each term, for each type of degree. We start with the Greek degree. According to Proposition 4.3, $\operatorname{deg}_{\gamma}(\Gamma u z)=-1$ and $\frac{\partial}{\partial(u z)}$ does not change the Greek degree. Moreover, for any $\nu$ that is a Greek variable, according to Proposition 3.11, $\Gamma \nu$ is a sum of terms all of Greek degree 0 , while $\partial / \partial \nu$ decreases the Greek degree by 1 . This proves that $\Gamma T$ is a sum of terms all of Greek degree $\operatorname{deg} \Gamma T=$ $\operatorname{deg}_{\gamma}(T)-1$.

We now treat $\operatorname{deg}_{+}$. We note that $\operatorname{deg}_{+}(\Gamma u z)=4$ and $\partial / \partial(u z)$ increases the pole degree $\operatorname{deg}_{+}$by 1 , thus the net effect of this term is 5 . For terms involving Greek variables, we 
observe that $\operatorname{deg}_{+}(\Gamma \zeta)=3$, and $\operatorname{deg}_{+}(\Gamma \eta)=5$, and their corresponding differentiation does not alter the pole degree $\operatorname{deg}_{+}$, resulting in a net effect of 5 . For $\zeta_{i}$, differentiation can decrease $\operatorname{deg}_{+}$by $2 i$ by removing a factor $\zeta_{i}$, but it is compensated by $\operatorname{deg}_{+}\left(\Gamma \zeta_{i}\right)=2 i+3$, which gives a net effect of at most 3 . For $\eta_{i}$, similarly to $\zeta_{i}$, the differentiation decreases $\operatorname{deg}_{+}$by $2 i$, but again $\operatorname{deg}_{+}\left(\Gamma \eta_{i}\right)=2 i+5$, giving a net effect of 5 . Combining all results, we have $\operatorname{deg}_{+}(\Gamma T) \leqslant \operatorname{deg}_{+}(T)+5$.

The case of $\mathrm{deg}_{-}$is addressed similarly. We observe that $\operatorname{deg}_{-}(\Gamma u z)=-2$ and $\partial / \partial(u z)$ increases the pole degree $\mathrm{deg}_{-}$by 1 , and the net effect of this term is -1 . For terms involving Greek variables, we observe that $\operatorname{deg}_{-}(\Gamma \zeta)=1$, and $\operatorname{deg}_{-}(\Gamma \eta)=-1$, while their corresponding differentiation has no effect on $\mathrm{deg}_{-}$, and the net effect is at most an increase by 1 . For $\eta_{i}$ and $\zeta_{i}$, their differentiation decreases deg- by $2 i$ by removing a factor $\eta_{i}$ or $\zeta_{i}$, but deg $\left(\Gamma \eta_{i}\right)=\operatorname{deg}_{-}\left(\Gamma \zeta_{i}\right)=2 i+1$, thus the net effect is also an increase by 1 . Therefore, $\operatorname{deg}_{-}(\Gamma T) \leqslant \operatorname{deg}_{-}(T)+1$.

Proof of Lemma 3.7. For $A \in \mathbb{Q}(u, z, \mathcal{G})$, since the operator $\Gamma$ is derivative, the quantity $\Gamma A$ is equal to

$$
\text { (Гu) } \frac{\partial}{\partial u} A+(\Gamma z) \frac{\partial}{\partial z} A+(\Gamma \zeta) \frac{\partial}{\partial \zeta} A+(\Gamma \gamma) \frac{\partial}{\partial \gamma} A+(\Gamma \eta) \frac{\partial}{\partial \eta} A+\sum_{i \geqslant 1}\left(\Gamma \eta_{i}\right) \frac{\partial}{\partial \eta_{i}} A+\sum_{i \geqslant 1}\left(\Gamma \zeta_{i}\right) \frac{\partial}{\partial \zeta_{i}} A .
$$

By Proposition 4.3 and Proposition 3.11, with the fact that $s=\frac{1-u z}{1+u z}$, we easily verify that $\Gamma A$ is also an element of $\mathbb{Q}(u, z, \mathcal{G})$. Moreover, if the poles of $A$ in $u$ are among $\pm \frac{1}{z}$, then so are the poles of $\Gamma A$. Note also that since $s$ has degree 0 in $u$, the quantity $\Gamma G$ for $G \in\{z\} \cup \mathcal{G}$ has degree 0 . Since $\Gamma u$ has degree 1 , and since differentiations decrease the degree by 1 , we conclude that the degree of $\Gamma A$ is at most the degree of $A$.

We now assume that $A$ is $u z$-symmetric. For $G \in\{z\} \cup \mathcal{G}$, the operator $\frac{\partial}{\partial G}$ preserves the $u z$-antisymmetry, and according to Proposition 4.3 and Proposition 3.11, $\Gamma G$ is $u z$-antisymmetric. Therefore, $(\Gamma G) \frac{\partial}{\partial G} A$ is $u z$-symmetric, being the product of two $u z$-antisymmetric factors. Moreover, by differentiating with respect to $u$ the expression $A(u)=-A\left(u^{-1} z^{-2}\right)$ we see that $\frac{u \partial}{\partial u} A$ is $u z$-symmetric. Since by Proposition $4.3, u^{-1} \Gamma u$ is $u z$-symmetric, this shows that $(\Gamma u) \frac{\partial}{\partial u} A$ is $u z$-symmetric, which concludes the proof that $\Gamma A$ is $u z$-symmetric.

\section{Structure of $Y(u)$ and expansion at the critical points.}

In this section we study the kernel $Y(u)$ at the points $u= \pm \frac{1}{z}$ via explicit computations. This is the place where we will see the Greek variables appear. The purpose of this section is to give the proofs of the propositions concerning $Y$, namely Proposition 3.4, Proposition 3.5 and Proposition 3.10. This will conclude the proof of all auxiliary results stated in the proof of Theorem 2.3.

\subsection{Structure of $Y(u)$ and proof of Proposition 3.4}

We can now proceed to a proof of Proposition 3.4 concerning the form of $Y$. 
Proof of Proposition 3.4. Writing

$$
\theta=\sum_{i=1}^{K} \frac{p_{i}}{x^{i}}=\sum_{i=1}^{K} \frac{p_{i}(1+u z)^{2 i}}{u^{i}}=(1+u z) \sum_{k=1}^{K} p_{k} z^{k} \sum_{\ell=0}^{2 k-1}(u z)^{\ell-k}\left(\begin{array}{c}
2 k-1 \\
\ell
\end{array}\right)
$$

and using the expression of $F_{0}$ in Proposition 3.2, one rewrites $2 F_{0}+\theta$ in the following form:

$$
2 F_{0}+\theta=(1+u z)\left(2-\sum_{k=1}^{K} p_{k} z^{k}\left(\sum_{\ell=1}^{k-1} u^{\ell} z^{\ell}\left(\begin{array}{c}
2 k-1 \\
k+\ell
\end{array}\right)-\sum_{\ell=-k}^{0} u^{\ell} z^{\ell}\left(\begin{array}{c}
2 k-1 \\
k+\ell
\end{array}\right)\right)\right) .
$$

We observe that $u^{K}\left(2 F_{0}+\theta\right)=(1+u z) Q(u)$ with $Q(u)$ polynomial in $u$ of degree $2 K-1$. The polynomial $Q(u)$ has the additional property that $\left[u^{k}\right] Q(u)$ is a polynomial in $z$, and for $k \geqslant K-1,\left[u^{k}\right] Q(u)$ is divisible by $z^{2(k-K)+1}$. We now evaluate $2 F_{0}+\theta$ at the point $u=1 / z$.

$$
\left.\left(2 F_{0}+\theta\right)\right|_{u=\frac{1}{z}}=4-2 \sum_{k=1}^{K} p_{k} z^{k}\left(\sum_{\ell=1}^{k-1}\left(\begin{array}{c}
2 k-1 \\
k+\ell
\end{array}\right)-\sum_{\ell=-k}^{0}\left(\begin{array}{c}
2 k-1 \\
k+\ell
\end{array}\right)\right)=4+4 \gamma .
$$

Therefore $\left.Q\right|_{u=\frac{1}{z}}=(2+2 \gamma) z^{-K}$. Now recall that $Y=1-x t\left(2 F_{0}+\theta\right)$ and that $x t=$ $\frac{u t}{(1+\gamma)(1+z u)^{2}}$, so we obtain:

$$
(1+u z)(1+\gamma) u^{K-1} Y=(1+u z)(1+\gamma) u^{K-1}-z Q(u) .
$$

When evaluated at $u=1 / z$, the right-hand side vanishes. This proves that the left-hand side, which is a polynomial in $u$ of degree $2 K-1$, has $(1-u z)$ as factor. We can thus write:

$$
Y=\frac{N(u)(1-u z)}{u^{K-1}(1+u z)(1+\gamma)}
$$

with $N(u)$ polynomial in $u$ of degree $2(K-1)$.

Proof of Proposition 3.5. We first observe that $Y^{2}$ is $u z$-symmetric. Indeed (using an idea already used in [3] and sometimes called the quadratic method, see e.g. [5]), we can rewrite the Tutte equation (8) for $g=0$ as follows:

$$
\left(1-x t\left(2 F_{0}+\theta\right)\right)^{2}=x^{2} t^{2} \theta^{2}-4 x t-2 x t \theta+1-4 x t\left(\Omega F_{0}-\theta F_{0}\right) .
$$

The right-hand is a Laurent polynomial in $x$, therefore it is symmetric. Since $Y=$ $1-x t\left(2 F_{0}+\theta\right)$, we conclude that $Y^{2}$ is symmetric. Since $Y$ is a Laurent polynomial in $z u$, it follows that $Y$ is either symmetric or antisymmetric, and to determine which, we examine its poles at $z u=0$ and $z u=\infty$. From the expression $Y=1-x t\left(2 F_{0}+\theta\right)$, from the definition of $\theta$, and from the explicit expression of $F_{0}$ given by Proposition 3.2, it is straightforward to check that:

$$
Y(u) \sim-t p_{k} /(z u)^{k-1} \text { when } z u \rightarrow 0, Y(u) \sim t p_{k}(z u)^{k-1} \text { when } z u \rightarrow \infty .
$$


We conclude that $Y$ is antisymmetric.

Now we study the zeros of $N(u)$. We will do this by studying the Newton polygon of $N(u)$, defined as the convex hull of the points $(i, j) \in \mathbb{R}^{2}$ such that the monomial $u^{i} z^{j}$ has non zero coefficient in $N(u)$.

We will rely on the computations done in the previous proof. We first observe that $\left[u^{K-1}\right]\left((1+u z)(1+\gamma) u^{K-1}-z Q(u)\right)$ is a polynomial in $z$ with a constant term 1 , therefore the same holds for $\left[u^{K-1}\right] N(u)$, which implies that the point $B=(K-1,0)$ is present in the Newton polygon of $N(u)$. Moreover, we observe that $\left[u^{0}\right]\left((1+u z)(1+\gamma) u^{K-1}-z Q(u)\right)=$ $-\left[u^{0}\right] z Q(u)$. But $\left[u^{0}\right] Q(u)=p_{K}$, therefore the point $A=(0,1)$ is present in the Newton polygon of $N(u)$. For any $k<K-1$, since $\left[u^{k}\right] Q(u)$ is a polynomial in $z$, the point $(k, 0)$ is never in the Newton polygon of $N(u)$. Therefore, the segment $A B$ is a side of the Newton polygon of $N(u)$, and accounts for the $(K-1)$ small roots of $N(u)$.

We then observe that $\left[u^{2 K-1}\right]\left((1+u z)(1+\gamma) u^{K-1}-z Q(u)\right)=-z\left[u^{2 K-1}\right] Q(u)=p_{K} z^{2 K}$. Therefore, the point $C=(2(K-1), 2 K-1)$ is present in the Newton polygon of $N(u)$. Furthermore, for any $k>K-1,\left[u^{k}\right]\left((1+u z)(1+\gamma) u^{K-1}-z Q(u)\right)=-z\left[u^{k}\right] Q(u)$, and $\left[u^{k}\right] Q(u)$ is divisible by $z^{k-K+1}$, thus $\left[u^{k}\right] N(u)$ is divisible by $z^{2(k-K)+2}$. The point corresponding to this term is $(k, 2(k-K)+2)$, and it always above the segment $B C$. We conclude that $B C$ is a side of the Newton polygon of $N(u)$, which accounts for the $(K-1)$ large roots of $N(u)$.

It remains to prove that the transformation $u \rightarrow \frac{1}{u z^{2}}$ exchanges large and small zeros of $N(u)$. Let $u_{0}$ be a small zero of $N(u)$, it is also a zero of $Y(u)$. But $Y$ is $u z$-antisymmetric, therefore $Y\left(u_{0}\right)=Y\left(u_{0}^{-1} z^{-2}\right)$, thus $u_{0}^{-1} z^{-2}$ is also a zero of $Y(u)$, and it is clearly not $1 / z$. The only possibility is that $u_{0}^{-1} z^{-2}$ is a zero of $N(u)$, and it is a large zero. Since the transformation $u \leftrightarrow u^{-1} z^{-2}$ is involutive, we conclude that it exchanges small and large zeros of $N(u)$.

\subsection{Expansion of $Y(u)$ and proof of Proposition 3.10}

We now study the expansion of $Y(u)$ at critical points. This is where Greek variables appear, and what explains their presence in Theorem 2.3.

We will start by the Taylor expansion of $2 F_{0}+\theta$. Since we are computing the Taylor expansion by successive differentiation by $u$, for simplicity, we will use the shorthand $\partial_{u}$ for $\frac{\partial}{\partial u}$. For integers $\ell$ and $a$, we define the falling factorial $(\ell)_{(a)}$ to be $(\ell)_{(a)}=$ $\ell(\ell-1) \ldots(\ell-a+1)$.

Proposition 5.1. At $u=1 / z$, we have the following Taylor expansion of $2 F_{0}+\theta$.

$$
2 F_{0}+\theta=4+4 \gamma-2(1-\eta)(1-u z)+\sum_{a \geqslant 2}(1-u z)^{a}\left((\eta+\gamma)+\sum_{i=1}^{\left\lfloor\frac{a-1}{2}\right\rfloor} c_{i, a}^{+} \eta_{i}\right)
$$

Here $c_{i, a}^{+}$are rational numbers depending only on $i, a$.

Proof. We proceed by computing successive derivatives evaluated at $u=1 / z$. In the proof of Proposition 3.4, we already showed that $\left(2 F_{0}+\theta\right)(u=1 / z)=4+4 \gamma$, which accounts for the first term. 
For other terms, using the expression of $2 F_{0}+\theta$ we used in the proof of Proposition 3.4 and grouping powers of $u z$ together we get

$$
\begin{aligned}
2 F_{0}+\theta & =(1+u z)\left(2-\sum_{k=1}^{K} p_{k} z^{k}\left(\sum_{\ell=1}^{k-1} u^{\ell} z^{\ell}\left(\begin{array}{c}
2 k-1 \\
k+\ell
\end{array}\right)-\sum_{\ell=-k}^{0} u^{\ell} z^{\ell}\left(\begin{array}{c}
2 k-1 \\
k+\ell
\end{array}\right)\right)\right) \\
& =(2+2 u z)+\sum_{k=1}^{K} p_{k} z^{k}\left(\sum_{\ell=-k}^{0} u^{\ell} z^{\ell}\left(\begin{array}{c}
2 k \\
k+\ell
\end{array}\right)-\sum_{\ell=2}^{k} u^{\ell} z^{\ell}\left(\begin{array}{c}
2 k \\
k+\ell
\end{array}\right)+\frac{2}{k+1}\left(\begin{array}{c}
2 k-1 \\
k
\end{array}\right) u z\right) .
\end{aligned}
$$

The first term is given by

$$
\begin{aligned}
\left.\partial_{u}\left(2 F_{0}+\theta\right)\right|_{u=1 / z} & =2 z+z \sum_{k=1}^{K} p_{k} z^{k}\left(\sum_{\ell=-k}^{0} \ell\left(\begin{array}{c}
2 k \\
k+\ell
\end{array}\right)-\sum_{\ell=2}^{k} \ell\left(\begin{array}{c}
2 k \\
k+\ell
\end{array}\right)+\frac{2}{k+1}\left(\begin{array}{c}
2 k-1 \\
k
\end{array}\right)\right) \\
& =2 z-z \sum_{k=1}^{K} p_{k} z^{k}(2 k-2)\left(\begin{array}{c}
2 k-1 \\
k
\end{array}\right)=2 z(1-\eta) .
\end{aligned}
$$

For any $a \geqslant 2$, the $a$-th differentiation of $2 F_{0}+\theta$ evaluated at $u=1 / z$ is

$$
\begin{aligned}
\left.\partial_{u}^{a}\left(2 F_{0}+\theta\right)\right|_{u=1 / z} & =z^{a} \sum_{k=1}^{K} p_{k} z^{k}\left(\sum_{\ell=-k}^{0}(\ell)_{(a)}\left(\begin{array}{c}
2 k \\
k+\ell
\end{array}\right)-\sum_{\ell=2}^{k}(\ell)_{(a)}\left(\begin{array}{c}
2 k \\
k+\ell
\end{array}\right)\right) \\
& =z^{a} \sum_{k=1}^{K} p_{k} z^{k}\left(\sum_{\ell=1}^{k}(-1)^{a}\left(\begin{array}{c}
2 k \\
k+\ell
\end{array}\right)(\ell+a-1)_{(a)}-\sum_{\ell=1}^{k}\left(\begin{array}{c}
2 k \\
k+\ell
\end{array}\right)(\ell)_{(a)}\right) .
\end{aligned}
$$

We are thus led to compute the quantity $\sum_{\ell=1}^{k}(\ell)_{(a)}\left(\begin{array}{c}2 k \\ k+\ell\end{array}\right)$ given $a \geqslant 2$ fixed for any $k$. It is natural to consider the following generating function:

$$
D_{a}(y)=\sum_{k \geqslant 0} y^{k} \sum_{\ell=1}^{k}(\ell)_{(a)}\left(\begin{array}{c}
2 k \\
k+\ell
\end{array}\right)=a ! \sum_{k \geqslant 0} y^{k} \sum_{\ell=1}^{k}\left(\begin{array}{l}
\ell \\
a
\end{array}\right)\left(\begin{array}{c}
2 k \\
k+\ell
\end{array}\right) .
$$

We choose to compute $D_{a}$ via a combinatorial interpretation in terms of lattice paths. Note that the number $\left[y^{k}\right] D_{a} / a$ ! counts paths of length $2 k$ with +1 and -1 steps, ending at height $2 \ell$ ( $k+\ell$ steps up and $k-\ell$ steps down), with $a$ distinct even and positive heights (including 0 ) below $2 \ell$ marked. By decomposing the whole path at the last passage for each height, we have the following equality.

$$
D_{a}(y)=a ! E(y)(1+C(y)) C(y)^{a} .
$$

Here, $E(y)$ is the generating function of paths ending at 0 , and $C(y)$ is the generating function of paths of even length ending in a strictly positive height. All these generating functions are classically expressed in terms of the one of Dyck paths as follows. Let $B(y)$ be the generating function of Dyck paths, i.e. paths ending at 0 and remaining always non-negative. We have by classical decompositions $E(y)=\frac{2}{1-(B(y)-1)}-1$ and 
$C(y)=\frac{y B(y)^{2}}{1-y B(y)^{2}}$. But we know that $B(y)$ verifies the equation $B(y)=1+y B(y)^{2}$, so we finally obtain the wanted expression of $D_{a}$ by substituting in (22) the expressions:

$$
B(y)=\frac{1-\sqrt{1-4 y}}{2 y}, E(y)=\frac{1}{\sqrt{1-4 y}}, C(y)=\frac{1}{2}\left(\frac{1}{\sqrt{1-4 y}}-1\right) .
$$

Similarly we now want to compute the quantity $\sum_{\ell=1}^{k}(\ell+a-1)_{(a)}\left(\begin{array}{c}2 k \\ k+\ell\end{array}\right)$ given $a \geqslant 2$ fixed for any $k$. We consider the following generating function:

$$
T_{a}(y)=\sum_{k \geqslant 0} y^{k} \sum_{\ell=1}^{k}(\ell+a-1)_{(a)}\left(\begin{array}{c}
2 k \\
k+\ell
\end{array}\right)=a ! \sum_{k \geqslant 0} y^{k} \sum_{\ell=1}^{k}\left(\begin{array}{c}
\ell+a-1 \\
a
\end{array}\right)\left(\begin{array}{c}
2 k \\
k+\ell
\end{array}\right)
$$

The combinatorial interpretation is essentially the same as $D_{a}(y)$, but in this case the $c$ heights are not necessarily distinct, therefore we have the following equality.

$$
T_{a}(y)=a ! E(y)(1+C(y))^{a} C(y) .
$$

We now observe that $\left.\left[p_{k} z^{k+a}\right] \partial_{u}^{a}\left(2 F_{0}+\theta\right)\right|_{u=1 / z}=\left[y^{k}\right]\left((-1)^{a} T_{a}(y)-D_{a}(y)\right)$, so the only generating function we need to consider is in fact $(-1)^{a} T_{a}(y)-D_{a}(y)$. Now,

$$
(-1)^{a} T_{a}(y)-D_{a}(y)=\frac{a !(-1)^{a}}{2^{a+1}} \frac{4 y}{(1-4 y)^{3 / 2}}\left(\left(\frac{1}{\sqrt{1-4 y}}+1\right)^{a-1}+\left(1-\frac{1}{\sqrt{1-4 y}}\right)^{a-1}\right) .
$$

We observe that, for any positive integer $a \geqslant 2$, the quantity $\frac{\sqrt{1-4 y}}{4 y}\left((-1)^{a} T_{a}(y)-D_{a}(y)\right)$ is a polynomial in $\frac{1}{1-4 y}$. More precisely expanding the $a-1$ powers we obtain:

$$
\begin{aligned}
\left.\partial_{u}^{a}\left(2 F_{0}+\theta\right)\right|_{u=1 / z} & =z^{a} \sum_{k=1}^{K} p_{k} z^{k}\left[y^{k}\right]\left((-1)^{a} T_{a}(y)-D_{a}(y)\right) \\
& =z^{a} \Theta^{-1}\left(\frac{a !(-1)^{a}}{2^{a}} s^{-2}\left(s^{-1}-s\right) \sum_{i=0}^{\left\lfloor\frac{a-1}{2}\right\rfloor}\left(\begin{array}{c}
a-1 \\
2 i
\end{array}\right) s^{-2 i}\right)
\end{aligned}
$$

We observe that $\Theta \eta_{1}=\frac{8}{3} s^{-3}\left(s^{-1}-s\right)^{2}$, and since $\Theta \eta_{i+1}=\left(s-s^{-1}\right) \partial_{s} \Theta \eta_{i}$, by induction on $i$ we know that $\Theta \eta_{i}$, as a Laurent polynomial in $s$, has a factor $\left(s-s^{-1}\right)^{2}$ for $i \geqslant$ 1. Therefore, from Proposition 4.1 we know that, for any polynomial $P\left(s^{-2}\right)$ in $s^{-2}$, $\Theta^{-1}\left(s^{-2}\left(s-s^{-1}\right) P\left(s^{-2}\right)\right)$ is a linear combination of $(\eta+\gamma)$ and $\eta_{i}$ for $i \geqslant 0$, and $[\eta+$ $\gamma] \Theta^{-1}\left(s^{-2}\left(s-s^{-1}\right) P\left(s^{-2}\right)\right)=4 P(1)$ by the fact that $\Theta(\eta+\gamma)=s^{-2}\left(s-s^{-1}\right) / 4$. We thus have

$$
\left.\partial_{u}^{a}\left(2 F_{0}+\theta\right)\right|_{u=1 / z}=a ! z^{a}(-1)^{a}\left((\eta+\gamma)+\sum_{i=1}^{\left\lfloor\frac{a-1}{2}\right\rfloor} c_{i, a}^{+} \eta_{i}\right)
$$

for some rational number $c_{i, a}^{+}$which concludes the proof. 
We now perform a very similar computation for the other pole $u=-1 / z$.

Proposition 5.2. At $u=-1 / z$, we have the following Taylor expansion of $2 F_{0}+\theta$.

$$
2 F_{0}+\theta=2(1+\zeta)(1+u z)+\sum_{a \geqslant 2}(1+u z)^{a}\left((\zeta-\gamma)+\sum_{i=1}^{\left\lfloor\frac{a-1}{2}\right\rfloor} c_{i, a}^{-} \zeta_{i}\right)
$$

Here $c_{i, a}^{-}$are rational numbers depending only on $i, a$.

Proof. For the constant term, we first note that from the expression used in the beginning of the proof of Proposition 3.4, it is obvious that $\left.\left(2 F_{0}+\theta\right)\right|_{u=-1 / z}$ vanishes.

We now treat higher-order terms. Using the last expression of $2 F_{0}+\theta$ in the proof of Proposition 5.1, the first order term is given by

$$
\begin{aligned}
& \left.\partial_{u}\left(2 F_{0}+\theta\right)\right|_{u=-1 / z} \\
& =2 z-z \sum_{k=1}^{K} p_{k} z^{k}\left(\sum_{\ell=-k}^{0}(-1)^{\ell} \ell\left(\begin{array}{c}
2 k \\
k+\ell
\end{array}\right)-\sum_{\ell=2}^{k}(-1)^{\ell} \ell\left(\begin{array}{c}
2 k \\
k+\ell
\end{array}\right)-\frac{2}{k+1}\left(\begin{array}{c}
2 k-1 \\
k
\end{array}\right)\right) \\
& =2 z-z \sum_{k=1}^{K} p_{k} z^{k} \frac{2-2 k}{2 k-1}\left(\begin{array}{c}
2 k-1 \\
k
\end{array}\right)=2 z(1+\zeta) .
\end{aligned}
$$

For any $a \geqslant 2$, the $a$-th differentiation of $2 F_{0}+\theta$ evaluated at $u=-1 / z$ is

$$
\begin{aligned}
& \left.\partial_{u}^{a}\left(2 F_{0}+\theta\right)\right|_{u=-1 / z} \\
& =z^{a} \sum_{k=1}^{K} p_{k} z^{k}\left(\sum_{\ell=1}^{k}(-1)^{\ell}\left(\begin{array}{c}
2 k \\
k+\ell
\end{array}\right)(\ell+a-1)_{(a)}-\sum_{\ell=1}^{k}(-1)^{\ell-a}\left(\begin{array}{c}
2 k \\
k+\ell
\end{array}\right)(\ell)_{(a)}\right) .
\end{aligned}
$$

We will now much borrow the combinatorial interpretation presented in the proof of Proposition 5.1. We now consider the following generating functions.

$$
\tilde{D}_{a}(y)=\sum_{k \geqslant 0} y^{k} \sum_{\ell=1}^{k}(-1)^{\ell}(\ell)_{(a)}\left(\begin{array}{c}
2 k \\
k+\ell
\end{array}\right) \quad, \quad \tilde{T}_{a}(y)=\sum_{k \geqslant 0} y^{k} \sum_{\ell=1}^{k}(-1)^{\ell}(\ell+a-1)_{(a)}\left(\begin{array}{c}
2 k \\
k+\ell
\end{array}\right) .
$$

We can see that $\left.\left[p_{k} z^{k+a}\right] \partial_{u}^{a}\left(2 F_{0}+\theta\right)\right|_{u=-1 / z}=\left[y^{k}\right]\left(\tilde{T}_{a}(y)-(-1)^{a} \tilde{D}_{a}(y)\right)$. Furthermore, these two generating functions have a combinatorial interpretation similar with $D_{a}(y)$ and $T_{a}(y)$ in the proof of Proposition 5.1, with the only difference that the parity of the height at the end also contributes as a sign. We define $\tilde{C}(y)=\frac{-y B(y)^{2}}{1+y B(y)^{2}}=\frac{1}{2}(\sqrt{1-4 y}-1)$. We have the following equalities, with $C(y)$ and $E(y)$ borrowed from the proof of Proposition 5.1.

$$
\tilde{D}_{a}(y)=a ! E(y)(1+\tilde{C}(y)) C(y)^{a}, \quad \tilde{T}_{a}(y)=a ! E(y)(1+\tilde{C}(y))^{a} C(y) .
$$


As in the previous proof we observe that for any $a \geqslant 2$, the quantity $\frac{\sqrt{1-4 y}}{4 y}\left(\tilde{T}_{a}(y)-\right.$ $\left.(-1)^{a} \tilde{D}_{a}(y)\right)$ is a polynomial in $(1-4 y)$. We also note that $\left[y^{k}\right] \frac{-4 y}{\sqrt{1-4 y}}(1-4 y)^{i}=$ $\left[x^{k} z^{k}\right] \frac{-4 x z}{\sqrt{1-4 x z}}(1-4 x z)^{i}$. Performing the computations explicitly we obtain as in the previous proof

$$
\left.\partial_{u}^{a}\left(2 F_{0}+\theta\right)\right|_{u=-1 / z}=\frac{z^{a} a !}{2^{a}} \Theta^{-1}\left(\left(s-s^{-1}\right) \sum_{i=0}^{\left\lfloor\frac{a-1}{2}\right\rfloor}\left(\begin{array}{c}
a-1 \\
2 i
\end{array}\right) s^{2 i}\right) .
$$

Finally, we observe that $\Theta(\zeta-\gamma)=\left(s-s^{-1}\right) / 4$, and $\Theta \zeta_{i}=\left(s^{-1}-s\right)\left(s^{2}-1\right)^{i}$, therefore, for any polynomial $P, \Theta^{-1}\left(\left(s-s^{-1}\right) P\left(s^{2}\right)\right)$ is a linear combination of $\zeta-\gamma$ and $\zeta_{i}$, and $[\zeta-\gamma] \Theta^{-1}\left(\left(s-s^{-1}\right) P\left(s^{2}\right)\right)=4 P(1)$. We thus have

$$
\left.\partial_{u}^{a}\left(2 F_{0}+\theta\right)\right|_{u=-1 / z}=z^{a} a !\left((\zeta-\gamma)+\sum_{i=1}^{\left\lfloor\frac{a-1}{2}\right\rfloor} c_{i, a}^{-} \zeta_{i}\right)
$$

for some rational numbers $c_{i, a}^{-}$.

The reader familiar with bijective techniques of map enumeration will notice that the paths appearing in the two previous proofs are of the same nature as the "label-walks" encountered around the unlabelled vertices of a mobile [7, 9]. This coincidence motivates the combinatorial approach that we have used to perform the computations in these proofs, and suggests that the occurrence of the derivatives of $Y(u)$ at the critical points has a combinatorial explanation in terms of mobiles. This question, that we leave open, certainly deserves to be better understood. We can now prove Proposition 3.10

Proof of Proposition 3.10. We just rewrite $x t P / Y$ as

$$
\frac{x t P}{Y}=\frac{1-u z}{1+u z} \frac{1}{(1+\gamma) \frac{(1+u z)^{2}}{u z}-\left(2 F_{0}+\theta\right)}
$$

and substitute the Taylor expansions of $2 F_{0}+\theta$ at $u= \pm 1 / z$ given by Propositions 5.2 and 5.1.

At this point, we have finished the proof Theorem 2.3 (including all the intermediate results that had been stated in Section 2). It remains to prove Theorem 2.1 and Theorem 2.2, which will be the purpose of the next section.

\section{Unrooting step and proof of Theorems 2.1 and 2.2}

In this section, we deduce Theorem 2.1 from Theorem 2.3, and we also check the exceptional case of genus 1 given by Theorem 2.2. Since the series $L_{g}$ and $F_{g}$ are related by the formula $F_{g}=\Gamma L_{g}$, studying $L_{g}$ from $L_{g}$ essentially amounts to inverting the differential 
operator $\Gamma$, i.e., heuristically, to perform some kind of integration. Since in our case the generating functions of rooted maps given by Theorem 2.3 are rational in our given set of parameters, it is no surprise that an important part of the work will be to show that this integration gives rise to no logarithm. This section is divided in two steps: we first construct (Section 6.1) two operators that enable us to "partially" invert the operator $\Gamma$ (Proposition 6.1), and we reduce the inversion of the operator $\Gamma$ to the computation of a univariate integral. Then (Section 6.3) we conclude the proof of Theorem 2.1 by proving that this integral contains no logarithms, using the combination of two combinatorial arguments: a disymmetry-type theorem, and an algebraicity statement proved with bijective tools in [9].

\subsection{The operators $\diamond$ and}

The first idea of the proof is inspired from [16], and consists of inverting the operator $\Gamma$ in two steps. We define the ring $\mathbb{L}$ formed by elements $f$ of $\mathbb{Q}\left[p_{1}, p_{2}, \ldots\right][[z]]$ such that for all $k \geqslant 0$, the coefficient of $z^{k}$ in $f$ is a homogeneous polynomial in the $p_{i}$ of degree $k$ (where the degree of $p_{i}$ is defined to be $i$ ). Equivalently, $\mathbb{L}=\mathbb{Q}\left[\left[z p_{1}, z^{2} p_{2}, z^{3} p_{3}, \ldots\right]\right]$. Note that any formal power series in the Greek variables, considered as an element of $\mathbb{Q}\left[p_{1}, p_{2}, \ldots\right][[z]]$, is an element of $\mathbb{L}$. Note also that $L_{g}$ is an element of $\mathbb{L}$. Indeed, if we view $L_{g}$ as a series in $t$, the coefficient of $t^{k}$ for $k \geqslant 0$ is a homogeneous polynomial of degree $k$ in the $p_{i}$, since the sum of half-face degrees in a bipartite map is equal to the number of edges. Given the form of the change of variable $t \leftrightarrow z$ given by (2), namely $t=z\left(1+\sum_{k}\left(\begin{array}{c}2 k-1 \\ l\end{array}\right) p_{k} z^{k}\right)^{-1}$, this clearly implies that as a series in $z, L_{g}$ is in $\mathbb{L}$.

We now introduce the linear operators $\square$ and $\diamond$ on $\mathbb{Q}\left[x, p_{1}, p_{2}, \ldots\right][[z]]$ defined by

$$
\square x^{k}=\left(\frac{1}{k}-\frac{\gamma}{1+\gamma}\right) p_{k}, \quad \diamond=\sum_{k} p_{k} \partial_{p_{k}},
$$

where $\partial_{p_{k}}$ is the differential operator defined by (16) in Section 4.1. We have:

Proposition 6.1. For any $A \in \mathbb{L}$, we have

$$
\diamond A=\square \Gamma A .
$$

In particular, $\diamond L_{g}=\square F_{g}$.

Proof. The proof is mainly a careful application of the chain rule and of the computations already made in Section 4 . Let $R \in \mathbb{L}$. Since $\Gamma$ is a derivation we have:

$$
\Gamma R=(\Gamma z) \frac{\partial}{\partial z} R+\sum_{k}\left(\Gamma p_{k}\right) \partial_{p_{k}} R=(\Gamma z) \frac{\partial}{\partial z} R+\sum_{k \geqslant 1} k x^{k} \partial_{p_{k}} R
$$

so that $\sum_{k} k x^{k} \partial_{p_{k}} R=\left(\Gamma-(\Gamma z) \frac{\partial}{\partial z}\right) R$. We now define the linear operators $\Pi: x^{k} \mapsto p_{k}$, and $\Xi: x^{k} \mapsto \frac{p_{k}}{k}$. By applying $\Xi$ to the last equality, we get:

$$
\sum_{k} p_{k} \partial_{p_{k}} R=\Xi\left(\Gamma-(\Gamma z) \frac{\partial}{\partial z}\right) R=\Xi \Gamma R-\Xi(\Gamma z) \frac{\partial}{\partial z} R .
$$


We thus need to study the expression of $\Xi(\Gamma z) \frac{\partial}{\partial z} R$. We notice that, over the ring $\mathbb{L}$, the operators $\Pi \sum_{k \geqslant 1} k \partial_{p_{k}}$ and $\frac{z \partial}{\partial z}$ are equal. Moreover, since $\Gamma z=\frac{\left(s^{-1}-s\right) z}{4 s^{2}(1-\eta)}$ by Proposition 3.11 , the operator $(\Gamma z) \frac{d}{d z}$ stabilises $\mathbb{L}$, so we have:

$$
\Pi \Gamma R=\Pi(\Gamma z) \frac{\partial}{\partial z} R+\Pi \sum_{k \geqslant 1} k x^{k} \partial_{p_{k}} R=\left(\Pi\left(\frac{\Gamma z}{z}\right)+1\right) z \frac{\partial}{\partial z} R=\frac{1+\gamma}{1-\eta} \frac{z \partial}{\partial z} R .
$$

(this is the only point in the proof where we use the assumption that $R \in \mathbb{L}$ ). Note that we have used that $\left(\Pi\left(\frac{\Gamma z}{z}\right)+1\right)=\left(\frac{1}{z} \sum_{k} k p_{k} \frac{\partial}{\partial p_{k}} z\right)+1=\frac{1+\gamma}{1-\eta}$ where the first equality comes from the definition of $\Pi$ and $\Gamma$, while the second follows from Proposition 4.2 and the definitions of $\gamma$ and $\eta$. The last displayed equation thus implies that:

$$
\frac{z \partial}{\partial z} R=\frac{1-\eta}{1+\gamma} \Pi \Gamma R
$$

Substituting this in the previous expression of $\sum_{k \geqslant 1} p_{k} \partial_{p_{k} R}$ and recalling $\Gamma z=\frac{\left(s^{-1}-s\right) z}{4 s^{2}(1-\eta)}$ we obtain:

$$
\begin{aligned}
\sum_{k \geqslant 1} p_{k} \partial_{p_{k}} R & =\Xi \Gamma R-\Xi\left(\frac{s^{-1}-s}{4 s^{2}(1+\gamma)}\right)(\Pi \Gamma) R \\
& =\Xi \Gamma R-\frac{\gamma}{(1+\gamma)}(\Pi \Gamma) R \\
& =\square \Gamma R,
\end{aligned}
$$

where the last equality is straightforward from the definitions of $\square, \Pi$, and $\Xi$, while the second one follows from the fact that $\Xi \frac{s^{-1}-s}{s^{2}}=D^{-1} \Theta^{-1} \gamma \frac{s^{-1}-s}{s^{2}} \gamma$, from Proposition 4.1 and a direct computation. This concludes the proof that $\diamond R=\square \Gamma R$ for $R \in \mathbb{L}$.

Finally, since $F_{g}=\Gamma L_{g}$ and $L_{g} \in \mathbb{L}$, it follows that $\diamond L_{g}=\square F_{g}$.

Proposition 6.2. $\diamond L_{g}$ is a rational function of the Greek variables, i.e.: $\diamond L_{g}=R$ with $R \in \mathbb{Q}[\mathcal{G}]$, whose denominator is of the form $(1-\eta)^{a}(1+\zeta)^{b}(1+\gamma)^{c}$ for $a, b \geqslant 0$ and $c \in\{0,1\}$.

Proof. We are going to use Theorem 2.3 and the fact that $\diamond L_{g}=\square F_{g}$. By Theorem 2.3, and since $F_{g}$ is $u z$-antisymmetric, we know that $F_{g}$ is an element of $\left(s^{-1}-s\right) \mathbb{Q}(\mathcal{G})\left[s^{2}, s^{-2}\right]$, where, as before, $s=\frac{1-u z}{1+u z}$. Therefore, we can write:

$$
F_{g}=\sum_{i \in I}\left(s^{-1}-s\right) s^{2 i} R_{i}
$$

where $I \subset \mathbb{Z}$ a finite set of integers and $R_{i} \in \mathbb{Q}(\mathcal{G})$ is a rational function in the Greek variables for each $i \in I$. Since $\diamond L_{g}=\square F_{g}$ we thus have:

$$
\diamond L_{g}=\sum_{i \in I} R_{i} \square\left(\left(s^{-1}-s\right) s^{2 i}\right) .
$$


Now, by Proposition 4.1, the vector space $\left(s^{-1}-s\right) \mathbb{Q}\left[s^{-2}, s^{2}\right]$ is spanned by the basis $B=\left\{\Theta \zeta_{i}, i \geqslant 1 ; \Theta(\zeta-\gamma) ; \Theta(\eta+\gamma) ; \Theta \eta_{i}, i \geqslant 1\right\}$. Moreover, the action of $\Theta$ on the basis $B$ is given by the formulas:

$$
\square \Theta \zeta_{i}=X_{i}-\frac{\gamma \zeta_{i}}{1+\gamma}, \square \Theta(\zeta-\gamma)=\zeta+\frac{\zeta-\gamma}{1+\gamma}, \square \Theta(\eta+\gamma)=\frac{\gamma(1-\eta)}{1+\gamma}, \square \Theta \eta_{i}=\eta_{i-1}-\frac{\gamma \eta_{i}}{1+\gamma},
$$

where $X_{i}$ is a linear combination of $\zeta, \zeta_{1}, \zeta_{2} \ldots, \zeta_{i}$ with rational coefficients. These formulas follow from the fact that $\square \Theta: p_{k} z^{k} \longmapsto\left(\frac{1}{k}-\frac{\gamma}{1+\gamma}\right) p_{k} z^{k}$ and from the definitions of Greek variables given in the statement of Theorem 2.1. Returning to (23), this proves that $\diamond L_{g}$ is a rational function of the Greek variables, $L_{g} \in \mathbb{Q}[\mathcal{G}]$. Finally, the form of the denominator is clear from the proof.

\subsection{Inverting $\diamond$}

Let $S \in \mathbb{Q}(\mathcal{G})$ be a rational function in the Greek variables, depending on a finite number of Greek variables. Since each Greek variable is a linear function of the $p_{k}$, it is clear that $\diamond$ leaves each Greek variable invariant. Since moreover, $\diamond$ is a derivation, this implies that $\diamond S$ is given by a simple univariate derivation:

$$
\diamond S=\left(\frac{d}{d v} S\left(v \eta, v \gamma,\left(v \eta_{i}\right)_{i \geqslant 1},\left(v \zeta_{i}\right)_{i \geqslant 1}\right)\right)_{v=1} .
$$

This implies:

Proposition 6.3. The series $L_{g}$ is given by:

$$
L_{g}=\int_{0}^{1} d v R\left(v \eta, v \gamma, v \zeta,\left(v \eta_{i}\right)_{i \geqslant 1},\left(v \zeta_{i}\right)_{i \geqslant 1}\right)
$$

where $R$ is the rational function such that $\diamond L_{g}=\square F_{g}=R\left(\eta, \gamma, \zeta,\left(\eta_{i}\right)_{i \geqslant 1},\left(\zeta_{i}\right)_{i \geqslant 1}\right)$.

Proof. We simply integrate (24). The only thing to check is the initial condition, namely that $R=0$ when all Greek variables are equal to zero. This is clear, since this specialisation is equivalent to substituting $z=0$, and since for $g \geqslant 1$ there is no map with 0 edge.

Corollary 6.4. The series $L_{g}$ has the following form:

$$
L_{g}=R_{1}+R_{2} \log (1-\eta)+R_{3} \log (1+\zeta)+R_{4} \log (1+\gamma)
$$

where $R_{1}, R_{2}, R_{3}, R_{4}$ are rational functions in $\left(\eta, \gamma, \zeta,\left(\eta_{i}\right)_{i \geqslant 1},\left(\zeta_{i}\right)_{i \geqslant 1}\right)$ depending on finitely many Greek variables. Furthermore, the denominator of $R_{1}$ is of the form $(1-\eta)^{a}(1+\zeta)^{b}$ for $a, b \geqslant 0$.

Proof. This follows from the last two propositions. Note that $R_{1}$ has no pole at $\gamma=-1$ since $\diamond L_{g}$ has at most a simple pole from Proposition 6.2. 


\subsection{Algebraicity and proof of Theorem 2.1}

In order to prove Theorem 2.1 from Corollary 6.4, it suffices to show that $R_{2}=R_{3}=0$, i.e. that no logarithms appear during the integration procedure. In order to do that, it is enough to show that the series $L_{g}$ is algebraic. We will do this in this section, using a detour via more combinatorial arguments, and using an algebraicity statement proved with bijective methods in [9].

The following lemma is a variant for maps of genus $g$ of the "disymmetry theorem" classical in the enumeration of labelled trees (and much popularised in the book [4]; see also [10] for a use in the context of planar maps).

Lemma 6.5 (Disymmetry theorem for maps). Let $L_{g}^{\text {vertex }}, L_{g}^{\text {face }}, L_{g}^{\text {edge }}$ be the exponential generating function of labelled bipartite maps of genus $g$ with a marked vertex, a marked face, and marked edge, respectively, by the number of edges (variable $t$ ) and the number of faces of half-degree $i$ (variable $p_{i}$, for $i \geqslant 1$ ). Then one has:

$$
(2-2 g) L_{g}=L_{g}^{\text {vertex }}+L_{g}^{\text {face }}-L_{g}^{\text {edge }} .
$$

Proof. This is a straightforward consequence of Euler's formula.

Now we observe that for clear combinatorial reasons, $L_{g}^{\text {face }}$ and $L_{g}^{\text {edge }}$ can be obtained from $F_{g}$ as follows:

$$
L_{g}^{\text {face }}=\Xi F_{g}, \quad: L_{g}^{\text {edge }}=\Pi F_{g},
$$

where $\Xi: x^{k} \mapsto \frac{p_{k}}{k}$ and $\Pi: x^{k} \mapsto p_{k}$ are defined as in the previous section. This implies:

Lemma 6.6. $L_{g}^{\text {face }}$ and $L_{g}^{\text {edge }}$ are rational functions of $\eta, \gamma, \zeta,\left(\eta_{i}\right)_{i \geqslant 1},\left(\zeta_{i}\right)_{i \geqslant 1}$.

Proof. Given Theorem 2.3, it is enough to prove that $\Xi$ and $\Pi$ send $\left(s^{-1}-s\right) \mathbb{Q}\left[s^{-2}, s^{2}\right]$ to rational functions of Greek variables. But by Proposition 4.1, any element $F \in\left(s^{-1}-\right.$ $s) \mathbb{Q}\left[s^{-2}, s^{2}\right]$ is such that $F=\Theta G$ where $G$ is a (finite) linear combination of elements of the basis $B=\left\{\eta+\gamma ; \zeta-\gamma ; \eta_{i}, i \geqslant 1 ; \zeta_{i}, i \geqslant 1\right\}$. Now it is clear from the definitions that we have

$$
\Pi \Theta: p_{k} \mapsto p_{k} \quad, \quad \Xi \Theta: p_{k} \mapsto \frac{p_{k}}{k} .
$$

We have to check each of these two operators sends an element of $B$ to a linear combination of Greek variables. For the first one, this is obvious. For the second one, we first observe that from the definition of Greek variables we have from a simple check that $\Xi \Theta(\eta+\gamma)=\gamma$, $\Xi \Theta(\zeta-\gamma)=2 \zeta-\gamma$, and $\Xi \Theta\left(\eta_{i}\right)=\eta_{i-1}$ for $i \geqslant 1$ (with $\eta_{0}=\eta$ ). Finally, for $i \geqslant 2$, one similarly checks that there exist rational numbers $\alpha_{i}, \beta_{i}$ such that $\Xi \Theta \zeta_{i}=\alpha_{i} \zeta_{i}+\beta_{i} \Xi \Theta \zeta_{i-1}$ which is enough to conclude by induction, together with the base case $\Xi \Theta \zeta_{1}=1 / 3\left(2 \zeta_{1}-\right.$ $2 \gamma+4 \zeta)$.

We now need the following result.

Proposition $6.7([9])$. Fix $g \geqslant 1$ and $D \subset \mathbb{N}$ a finite subset of the integers of maximum at least 2 . Let $\mathbf{p}_{D}$ denote the substitution $p_{i}=\mathbf{1}_{i \in D}$ for $i \geqslant 1$. Then the series $L_{g}^{\text {vertex }}\left(\mathbf{p}_{D}\right)$ is algebraic, i.e there exists a nonzero polynomial $Q \in \mathbb{Q}[t ; X]$ such that $Q\left(t ; L_{g}^{\text {vertex }}\left(t ; \ldots p_{i}=\mathbf{1}_{i \in D} \ldots\right)\right)=0$. 
Proof. Since this statement is not written in this form in [9], let us clarify where it comes from. Let $O_{g} \equiv O_{g}\left(t ; p_{1}, p_{2}, \ldots\right)$ be the ordinary generating function of rooted bipartite maps with one pointed vertex, by the number of edges (variable $t$ ) and faces (variable $p_{i}$ for faces of half-degree $i$, including the root face). Then it is easy to see that we have $O_{g}=\frac{t d}{d t} L_{g}^{v e r t e x}$. Now in [9, Eq. (8.2)] (and more precisely in the case $m=2$ of that reference), it is proved that there exists an algebraic series $R_{D}=R_{D}(t)$ such that $R_{D}(t=0)=0$ and $O_{g}\left(\mathbf{p}_{D}\right)=\frac{t d}{d t} R_{D}$, where, as above, $O_{g}\left(\mathbf{p}_{D}\right)$ is the series $O_{g}$ under the substitution $p_{i}=\mathbf{1}_{i \in D}$. Since $L_{g}^{\text {vertex }}(t=0)=0$ for clear combinatorial reasons, we have $L_{g}^{\text {vertex }}\left(\mathbf{p}_{D}\right)=R_{D}$

We can now prove Theorem 2.1.

Proof of Theorem 2.1. For $g \geqslant 2$, we can conclude from Lemma 6.5, Lemma 6.6 and Proposition 6.7 that for any finite set $D$ of integers with maximum at least 2 the series $L_{g}\left(\mathbf{p}_{D}\right)$ is algebraic, where, as before, $\mathbf{p}_{D}$ denotes the substitution of variables $p_{i}=\mathbf{1}_{i \in D}$ for $i \geqslant 1$. This implies that the three rational functions $R_{2}, R_{3}$, and $R_{4}$ defined in Corollary 6.4, are vanishing under that specialisation:

$$
R_{2}\left(\mathbf{p}_{D}\right)=0 \quad ; \quad R_{3}\left(\mathbf{p}_{D}\right)=0 \quad ; \quad R_{4}\left(\mathbf{p}_{D}\right)=0
$$

Therefore to conclude the proof that $R_{2}=R_{3}=R_{4}=0$ it is enough to show that if $Q$ is a polynomial in the Greek variables, $Q \in \mathbb{Q}[\mathcal{G}]$, such that $Q\left(\mathbf{p}_{D}\right)=0$ for all finite $D$, then $Q=0$.

This can be proved by infinite descent on the Greek degree $\operatorname{deg}_{\gamma}$, where each Greek variable is of degree 1 . Let $Q$ be a non-zero element of $\mathbb{Q}[\mathcal{G}]$ with minimal Greek degree such that $Q\left(p_{D}\right)=0$ for all finite $D$. It is clear that $Q$ cannot be a constant, therefore $\operatorname{deg}_{\gamma}(Q) \geqslant 1$. We denote by $c$ the maximal index of all Greek variables $\eta_{i}$ and $\zeta_{i}$ that

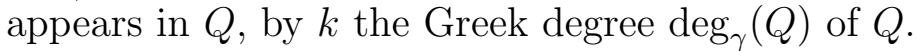

Let $D$ be a finite subset of $\mathbb{N}_{+}$, and $d$ its maximum. Let $\ell$ be an integer larger than $d$. We define $X=\left(\begin{array}{c}2 \ell-1 \\ \ell\end{array}\right)$, and we denote by $D^{*}=D \cup\{\ell\}$. We observe that, for any Greek variable $G \in \mathcal{G}$, we have

$$
G\left(p_{D^{*}}\right)=G\left(p_{D}\right)+R_{G}(\ell) X,
$$

where $R_{G}(\ell)$ is a rational function of $\ell$ that depends on the Greek variable $G$. We can thus write $Q\left(p_{D^{*}}\right)$ as

$$
Q\left(p_{D^{*}}\right)=\sum_{i=0}^{k} Q_{i}\left(\ell, p_{D}\right) X^{i},
$$

where the coefficients $Q_{i}\left(\ell, p_{D}\right)$ are rational in $\ell$ and polynomial in $G\left(p_{D}\right)$ for all Greek variables $G \in \mathcal{G}$. Furthermore, the total degree of $Q_{i}\left(\ell, p_{D}\right)$ in all Greek variable specializations $G\left(p_{D}\right)$ is at most $k-i$. When $\ell$ tends to infinity, $X$ grows exponentially with $\ell$, whereas each coefficient $Q_{i}\left(\ell, p_{D}\right)$ grows at most polynomially. Since $Q\left(p_{D^{*}}\right)=0$, this implies that for any $i$ and $D$ we must have $Q_{i}\left(\ell, p_{D}\right)=0$ for infinitely many values of $\ell$. 
We now consider $Q_{1}$ (which is well defined since $k \geqslant 1$ ). From its definition, we have the following expression:

$$
\begin{aligned}
Q_{1}\left(\ell, p_{D}\right)=\left[X^{1}\right] Q\left(p_{D^{*}}\right)= & \frac{\partial Q}{\partial \gamma}\left(p_{D}\right)+(\ell-1) \frac{\partial Q}{\partial \eta}\left(p_{D}\right)+\frac{\ell-1}{2 \ell-1} \frac{\partial Q}{\partial \zeta}\left(p_{D}\right)+\sum_{i=1}^{c} \ell^{i}(\ell-1) \frac{\partial Q}{\partial \eta_{i}}\left(p_{D}\right) \\
& +\sum_{i=1}^{c} \frac{(-2)^{i+1} \ell(\ell-1) \cdots(\ell-i)}{(2 \ell-1)(2 \ell-3) \cdots(2 \ell-2 i-1)} \frac{\partial Q}{\partial \zeta_{i}}\left(p_{D}\right)
\end{aligned}
$$

We fix the set $D$, and for an infinite number of values of $\ell$, we have $Q_{1}\left(\ell, p_{D}\right)=0$. Therefore, we must have $Q_{1}\left(\ell, p_{D}\right)=0$ as a rational fraction of $\ell$. However, since the rational fractions and polynomials in $\ell$ appearing in each term of the right-hand side of (25) are linearly independent, we must have $(\partial Q / \partial G)\left(p_{D}\right)=0$ for all Greek variables $G \in \mathcal{G}$. Since the equality holds for all finite set $D$, and since there is at least one Greek variable $G$ such that $\partial Q / \partial G$ is a non-zero polynomial, we thus have found a polynomial of lower Greek degree that vanishes under all specializations $p_{D}$, a contradiction. This concludes the proof that $R_{2}=R_{3}=R_{4}=0$.

We thus have proved the rationality of $L_{g}$ for $g \geqslant 2$, and by Corollary 6.4, the denominator of $L_{g}$ is of the form $(1-\eta)^{a}(1+\zeta)^{b}$ for $a, b \geqslant 0$.

We now prove that, expressed as a rational function in Greek variables, $L_{g}$ does not depend on $\gamma$. From the last paragraph we already now that $L_{g}$ is a polynomial in $\gamma$, i.e. $L_{g}=\sum_{i=0}^{k} S_{i} \gamma^{i}$ where $k \geqslant 0$, the $S_{i}$ are rational function of $\mathcal{G} \backslash\{\gamma\}$, and $S_{k} \neq 0$. Recall that $F_{g}=\Gamma L_{g}$, so from the fact that $\Gamma$ is a derivation and from the expressions (18)(21), $F_{g}$ is also polynomial in $\gamma$. Moreover, the only Greek variable $G \in \mathcal{G}$ such that $\Gamma G$ depends on $\gamma$ is $G=\gamma$, and we have more precisely $\Gamma \gamma=\frac{s^{-1}-s}{4(1-\eta) s^{2}}(\eta+\gamma)+\frac{1}{4}\left(s^{-3}-s^{-1}\right)$. It follows that the coefficient of $\gamma^{k}$ in $F_{g}$ is equal to $k\left(\Gamma S_{k}\right)+\frac{s^{-3}-s^{-1}}{4(1-\eta)} S_{k}$. From the structure of $F_{g}$ provided by Theorem $2.3\left(F_{g}\right.$, as a rational function in Greek variables and $u z$, does not depend on $\gamma$ ), this coefficient is equal to zero. It is easy to see that this is impossible if $k>0$. Indeed, looking at (18)-(21) again, $\Gamma S_{k}$ contains either a pole of order at least 5 at $u=1 / z$, or a pole of order at least 1 at $u=-1 / z$, which cannot be cancelled by the factor $\left(s^{-3}-s^{-1}\right)$ in the second term. Therefore $k=0$, i.e. $L_{g}$ does not depend of $\gamma$.

It only remains to prove the bound conditions. Recall that

$$
F_{g}=\Gamma L_{g}=(\Gamma \zeta) \frac{\partial}{\partial \zeta} L_{g}+(\Gamma \eta) \frac{\partial}{\partial \eta} L_{g}+\sum_{i \geqslant 1}\left(\Gamma \eta_{i}\right) \frac{\partial}{\partial \eta_{i}} L_{g}+\sum_{i \geqslant 1}\left(\Gamma \zeta_{i}\right) \frac{\partial}{\partial \zeta_{i}} L_{g}
$$

Using the three notions of degree in Section 3.3, we only need to check that $L_{g}$ is a homogeneous sum of Greek degree $\operatorname{deg}_{\gamma}\left(L_{g}\right)=2-2 g$ and $\operatorname{deg}_{+}\left(L_{g}\right)=\operatorname{deg}_{-}\left(L_{g}\right) \leqslant 6(g-1)$. For the Greek degree, we observe that, by Proposition 3.11 and the fact that $L_{g}$ has no constant term, if $L_{g}$ is not homogeneous in Greek degree, then $F_{g}=\Gamma L_{g}$ cannot be homogeneous. Therefore, $L_{g}$ must be homogeneous, with degree $\operatorname{deg}_{\gamma}\left(L_{g}\right)=\operatorname{deg}_{\gamma}\left(F_{g}\right)+$ $1=2-2 g$.

For the pole degree $\operatorname{deg}_{+}$, let $T=c \eta_{\alpha} \zeta_{\beta}(1-\eta)^{-a}(1+\zeta)^{-b}$ for $c \in \mathbb{Q}, a, b \geqslant 0$ and $\alpha, \beta$ two partitions be the largest term in $L_{g}$ such that $\operatorname{deg}_{+}(T)=\operatorname{deg}_{+}\left(L_{g}\right)$ when ordered first alphabetically by $\alpha$ then also alphabetically by $\beta$. We will now discuss by cases. 
If $\alpha$ and $\beta$ are both empty, then $\operatorname{deg}_{+}(T)=0$ and we are done.

We now suppose that $\alpha$ is empty but not $\beta$. We observe that, for a term $S$ in the form $c \zeta_{\beta^{\prime}}(1-\eta)^{-a}(1+\zeta)^{-b}$, if we order the terms in $\Gamma S$ first by the power of $(1+u z)$ in the denominator then alphabetically by $\nu$ in their factor of the form $\zeta_{\nu}$, then the largest term $S^{\prime}$ comes from $\left(\Gamma \zeta_{\beta_{1}^{\prime}}\right) \partial S / \partial \zeta_{\beta_{1}^{\prime}}$, with pole degree $\operatorname{deg}_{-}\left(S^{\prime}\right)=2\left|\beta^{\prime}\right|+1$ and no possibility of cancellation. Therefore, in $F_{g}$ there is a term $T^{\prime}$ coming from $\left(\Gamma \zeta_{\beta_{1}}\right) \partial T / \partial \zeta_{\beta_{1}}$ that can have no cancellation by the maximality of $\beta$ and by our previous observation, and $\operatorname{deg}_{-}\left(T^{\prime}\right)=2|\beta|+1$. But since $\operatorname{deg}_{-}\left(F_{g}\right) \leqslant 2 g-1$, we have $\operatorname{deg}_{-}\left(L_{g}\right)=2|\beta| \leqslant 2 g-2$, which concludes this case.

The final case is that $\alpha$ is nonempty. We observe that, for a term $S$ in the form $c \eta_{\alpha^{\prime}} \zeta_{\beta^{\prime}}(1-\eta)^{-a}(1+\zeta)^{-b}$, if we order the terms in $\Gamma S$ first by the power of $(1-u z)$ in the denominator then alphabetically by $\nu$ in their factor of the form $\eta_{\nu}$, then the largest term $S^{\prime}$ comes from $\left(\Gamma \eta_{\alpha_{1}^{\prime}}\right) \partial S / \partial \eta_{\alpha_{1}^{\prime}}$, with pole degree $\operatorname{deg}_{+}\left(S^{\prime}\right)=2\left|\alpha^{\prime}\right|+2\left|\beta^{\prime}\right|+5$ and no possibility of cancellation. Therefore, similarly to the previous case, by the fact that $\operatorname{deg}_{+}\left(F_{g}\right) \leqslant 6 g-1$, we conclude that $\operatorname{deg}_{+}\left(L_{g}\right)=2|\alpha|+2|\beta| \leqslant 6(g-1)$. We thus cover all cases and conclude the proof.

The only thing that remains now is to address the case of genus 1 :

Proof of Theorem 2.2. First note that since all the steps performed in the proof of Theorem 2.3, including the proofs of Propositions 5.1 and 5.2 are computationally effective, one can compute the value of $F_{1}$ using Theorem 3.9 for $g=1$ (the only "input" needed is the value of $F_{0}^{(2)}$ given by $(11)$ ). It then only remains to check that applying the operator $\Gamma$ to the expression of Theorem 2.2 gives back this expression, which is an automatic check given the computations of Section 4 (more reliably done using a computer algebra system).

Alternatively, note that the value of $F_{1}$ (resp. $L_{1}$ ) can be obtained by using the structure given by Theorem 2.3 (resp. by making explicit the degree bounds in the structure given by Corollary 6.4) and computing sufficiently many terms of the expression of $F_{1}$ (for example by iterating the Tutte equation (8)) to identify all undetermined coefficients appearing in these expressions. Again, these computations are automatic and more easily performed with a computer.

\section{$7 \quad$ Final comments}

We conclude this paper with several comments.

First, as explained in the introduction, we have only used two basic ideas from the topological recursion of [14]. It may be the case that other features of the latter can be applied to bipartite maps. This may provide a different way of performing the "unrooting" step performed in Section 6, similar to [13, Sec. III-4.2]. In particular this may give another way to derive the explicit expression of $L_{1}$, similarly to what has been done for other models satisfying the topological recursion (such as even-maps, see [13]). However the proof we gave has the nice advantage of providing a partly combinatorial explanation for the absence of logarithms in genus $g>1$. More generally, it seems that understanding 
the link between the disymmetry argument we used here and statements such as $[13$, Thm III 4.2] is an interesting question from the viewpoint of the topological recursion itself, and for the understanding of its combinatorial meaning.

Our next comment is about computational efficiency. While it is tempting to use Theorem 3.9 to compute the explicit expression of $F_{g}$ (and then $L_{g}$ ), it is much easier to simply compute the first few terms of $F_{g}$ (and $L_{g}$ ) using recursively the Tutte equation (8), and then determine the unknown coefficients in (4) or (5) by solving a linear system (recall that (4) and (5) are finite sums, so there are indeed finitely many coefficients to determine).

Third, structure results similar to Theorem 2.3 for the "multi-boundary" generating functions $F_{g}^{(m)}\left(x_{1}, x_{2}, \ldots, x_{m}\right)$ of bipartite maps of genus $g$ carrying $m \geqslant 1$ marked faces whose sizes are recorded in the exponents of variables $x_{1}, x_{2}, \ldots, x_{m}$, are easily derived from our results. Indeed, this series is obtained by applying $m$ times to $L_{g}$ the rooting operator $\Gamma$, one time in each variable. More precisely:

$$
F_{g}^{(m)}\left(x_{1}, x_{2}, \ldots, x_{m}\right)=\Gamma_{1} \Gamma_{2} \ldots \Gamma_{m} L_{g},
$$

where $\Gamma_{i}=\sum_{k \geqslant 1} k x_{i}^{k} \frac{\partial}{\partial p_{k}}$. Since the action of $\Gamma_{i}$ is fully described by Proposition 3.11 (up to replacing $s$ by $s_{i}=\frac{1-u_{i} z}{1+u_{i} z}$, where $u_{i}=x_{i}\left(1+z u_{i}\right)^{2}$ ), the series $F_{g}^{(m)}\left(x_{1}, x_{2}, \ldots, x_{m}\right.$ ) are easily computable rational functions in the Greek variables and the $\left(1 \pm u_{i} z\right)$. We observe as well that, by substituting all the $p_{i}$ to zero in the series $F_{g}^{(m)}\left(x_{1}, x_{2}, \ldots, x_{m}\right)$, one obtains the generating function for bipartite maps having exactly $m$ faces, where the $x_{i}$ control the face degrees. Therefore, these functions have a nice structure as well, being polynomials in the $1 /\left(1 \pm u_{i} z\right)$ with rational coefficients. This special case also follows from the results of [19]

Finally, it is natural to investigate further links between our results and those in $[18,16]$. One such link is provided by the topological recursion, which is related to all of them, but it seems that even stronger analogies hold between these models. For example, it is tempting to look for a general model encapsulating all these results. This is the subject of a work in progress.

\section{Acknowledgements}

Both authors thank Mireille Bousquet-Mélou for interesting discussions and comments.

\section{References}

[1] J. Ambjørn, L. Chekhov, and Yu. Makeenko. Higher genus correlators from the Hermitian one-matrix model. Phys. Lett. B, 282(3-4):341-348, 1992.

[2] E. A. Bender and E. R. Canfield. The number of rooted maps on an orientable surface. J. Combin. Theory Ser. B, 53(2):293-299, 1991.

[3] E. A. Bender and E. R. Canfield. The number of degree-restricted rooted maps on the sphere. SIAM J. Discrete Math., 7(1):9-15, 1994. 
[4] F. Bergeron, G. Labelle, and P. Leroux. Combinatorial species and tree-like structures, volume 67 of Encyclopedia of Mathematics and its Applications. Cambridge University Press, Cambridge, 1998. Translated from the 1994 French original by Margaret Readdy, With a foreword by Gian-Carlo Rota.

[5] M. Bousquet-Mélou. Rational and algebraic series in combinatorial enumeration. In International Congress of Mathematicians. Vol. III, pages 789-826. Eur. Math. Soc., Zürich, 2006.

[6] M. Bousquet-Mélou and A. Jehanne. Polynomial equations with one catalytic variable, algebraic series and map enumeration. J. Combin. Theory Ser. B, 96(5):623672, 2006.

[7] J. Bouttier, P. Di Francesco, and E. Guitter. Planar maps as labeled mobiles. Electron. J. Combin., 11(1):Research Paper 69, 27, 2004.

[8] S. R. Carrell and G. Chapuy. Simple recurrence formulas to count maps on orientable surfaces. J. Combin. Theory Ser A, 133:58-75, 2015. see also arXiv:1402.6300.

[9] G. Chapuy. Asymptotic enumeration of constellations and related families of maps on orientable surfaces. Combin. Probab. Comput., 18(4):477-516, 2009.

[10] G. Chapuy, É. Fusy, M. Kang, and B. Shoilekova. A complete grammar for decomposing a family of graphs into 3-connected components. Electron. J. Combin., 15(1):Research Paper 148, 39, 2008.

[11] G. Collet and É. Fusy. A simple formula for the series of constellations and quasiconstellations with boundaries. Electron. J. Combin., 21(2):Paper 2.9, 27, 2014.

[12] T. Ekedahl, S. Lando, M. Shapiro, and A. Vainshtein. Hurwitz numbers and intersections on moduli spaces of curves. Invent. Math., 146(2):297-327, 2001.

[13] B. Eynard. Counting surfaces, volume 70 of Progress in Mathematical Physics. Birkhäuser/Springer, 2016. CRM Aisenstadt chair lectures.

[14] B. Eynard and N. Orantin. Topological recursion in enumerative geometry and random matrices. J. Phys. A, 42(29):293001, 117, 2009.

[15] Z. Gao. The number of degree restricted maps on general surfaces. Discrete Math., 123(1-3):47-63, 1993.

[16] I. P. Goulden, M. Guay-Paquet, and J. Novak. Polynomiality of monotone Hurwitz numbers in higher genera. Adv. Math., 238:1-23, 2013.

[17] I. P. Goulden and D. M. Jackson. The KP hierarchy, branched covers, and triangulations. Adv. Math., 219(3):932-951, 2008.

[18] I. P. Goulden, D. M. Jackson, and R. Vakil. The Gromov-Witten potential of a point, Hurwitz numbers, and Hodge integrals. Proc. London Math. Soc. (3), 83(3):563-581, 2001.

[19] M. Kazarian and P. Zograf. Virasoro constraints and topological recursion for Grothendieck's dessin counting. Lett. Math. Phys., 105(8):1057-1084, 2015. 
[20] S. K. Lando and A. K. Zvonkin. Graphs on surfaces and their applications, volume 141 of Encyclopaedia of Mathematical Sciences. Springer-Verlag, 2004. Appendix by D. B. Zagier.

[21] G. Schaeffer. Planar maps. In Handbook of enumerative combinatorics, Discrete Math. Appl. (Boca Raton), pages 335-395. CRC Press, Boca Raton, FL, 2015.

[22] W. T. Tutte. A census of planar maps. Canad. J. Math., 15:249-271, 1963.

[23] T. R. S. Walsh and A. B. Lehman. Counting rooted maps by genus. I. J. Combinatorial Theory Ser. B, 13:192-218, 1972. 